Virginia Commonwealth University vCU Scholars Compass

2014

\title{
Temperature dependence of defect-related photoluminescence in III-V and II-VI semiconductors
}

Michael A. Reshchikov

Virginia Commonwealth University, mreshchi@vcu.edu

Follow this and additional works at: http://scholarscompass.vcu.edu/phys_pubs

Part of the Physics Commons

Copyright (C) 2014 AIP Publishing LLC. Reuse of AIP content is subject to the terms at: http://scitation.aip.org/ termsconditions. Originally published by the American Institute of Physics at http://dx.doi.org/10.1063/1.4838038.

\section{Downloaded from}

http://scholarscompass.vcu.edu/phys_pubs/4

This Article is brought to you for free and open access by the Dept. of Physics at VCU Scholars Compass. It has been accepted for inclusion in Physics Publications by an authorized administrator of VCU Scholars Compass. For more information, please contact libcompass@vcu.edu. 


\title{
Temperature dependence of defect-related photoluminescence in III-V and II-VI semiconductors
}

\author{
Michael A. Reshchikova) \\ Department of Physics, Virginia Commonwealth University, Richmond, Virginia 23284, USA
}

(Received 25 July 2013; accepted 20 September 2013; published online 2 January 2014)

\begin{abstract}
Mechanisms of thermal quenching of photoluminescence (PL) related to defects in semiconductors are analyzed. We conclude that the Schön-Klasens (multi-center) mechanism of the thermal quenching of PL is much more common for defects in III-V and II-VI semiconductors as compared to the Seitz-Mott (one-center) mechanism. The temperature dependencies of PL are simulated with a phenomenological model. In its simplest version, three types of defects are included: a shallow donor, an acceptor responsible for the PL, and a nonradiative center that has the highest recombination efficiency. The case of abrupt and tunable thermal quenching of PL is considered in more detail. This phenomenon is predicted to occur in high-resistivity semiconductors. It is caused by a sudden redirection of the recombination flow from a radiative acceptor to a nonradiative defect. (C) 2014 AIP Publishing LLC. [http://dx.doi.org/10.1063/1.4838038]
\end{abstract}

\section{INTRODUCTION}

Photoluminescence (PL) is a powerful tool used to study point defects in semiconductors. In particular, the temperature dependence of PL intensity, band shape and position provides valuable information about the defects and helps to identify them. At very low temperatures $(T<15 \mathrm{~K})$, excitonic lines in high-quality direct bandgap semiconductors are narrow, whereas defect-related bands are commonly broad because of the strong electron-phonon coupling typical for deep-level defects. With increasing temperature, the broad PL bands just slightly change their shape and position. However, the intensity of the PL, $I^{P L}$, may change by orders of magnitude. The internal quantum efficiency (IQE) of the defect-related PL, $\eta=I^{P L} / G$, where $G$ is the electron-hole pair generation rate, typically has the following dependence on temperature: ${ }^{1}$

$$
\eta(T)=\frac{\eta_{0}}{1+C \exp \left(-\frac{E_{A}}{k T}\right)} .
$$

This dependence consists of two parts: a temperatureindependent part at $T<T_{0}$ and an exponential decrease (called the PL quenching) at $T>T_{0}$, where $T_{0}$ can be found from the relation $C \cdot \exp \left(-E_{A} / k T_{0}\right)=1$. This quenching of a defect-related PL in an $n$-type semiconductor is commonly attributed to one of the following mechanisms that can be explained with the help of an energy band diagram or in terms of the one-dimensional configuration coordinate diagram, ${ }^{2,3}$ as shown in Fig. 1.

According to the first mechanism, PL quenching in an $n$-type semiconductor is due to the escape of holes from a defect (usually an acceptor) level to the valence band which begins at a critical temperature $T_{0}$, above which the PL quenching takes place with the activation energy equal to the

\footnotetext{
${ }^{a)}$ Electronic mail: mreshchi@vcu.edu.
}

ionization energy of this defect, $E_{A 1}$. In this mechanism, the thermally emitted holes (transition 4 in Fig. 1) may be captured back by the defect (transition 2) or recombine with electrons via other recombination channels (not shown in Fig. 1). Since other types of defects are needed for the explanation, we will call this mechanism the multi-center model of PL quenching. It was originally proposed by Schön and Klasens. ${ }^{4,5}$ They suggested that nonradiative transitions, associated with other defect centers (called the killer centers or poisons) result in the thermal quenching of PL from radiative defects. The PL quenching in this model can be described with Eq. (1) in which ${ }^{1}$

$$
C=\left(1-\eta_{0}\right) \tau_{P L} C_{p A} \frac{N_{v}}{g} \quad \text { and } \quad E_{A}=E_{A 1}
$$

where $\tau_{P L}$ is the PL lifetime, $\eta_{0}$ is the IQE of this PL at low temperatures before the quenching, $C_{p A}$ is the hole-capture coefficient for the defect, $N_{v}$ is the effective density of states in the valence band, and $g$ is the degeneracy factor of the defect. This is the most common mechanism of PL quenching for defects in $n$-type GaN where the majority of the defect-related PL bands are caused by transitions from shallow donors or from the conduction band to different acceptors. $^{6}$

According to the second mechanism, PL quenching is caused by the gradual replacement of radiative transitions by non-radiative ones involving the same defect (one-center model). It was first proposed by Seitz ${ }^{7}$ and Mott. ${ }^{8}$ This model can be better explained by using the configuration coordinate diagram shown in Fig. 1(b). It involves the temperatureinduced excitation of the defect to the level where adiabatic potentials of its ground and excited states cross, followed by a nonradiative recombination of the bound hole with the free electron and the emission of a large number of phonons (transition 5). ${ }^{2,3}$ In this case, the activation energy of the PL quenching is equal to the energy difference between the 
(a)

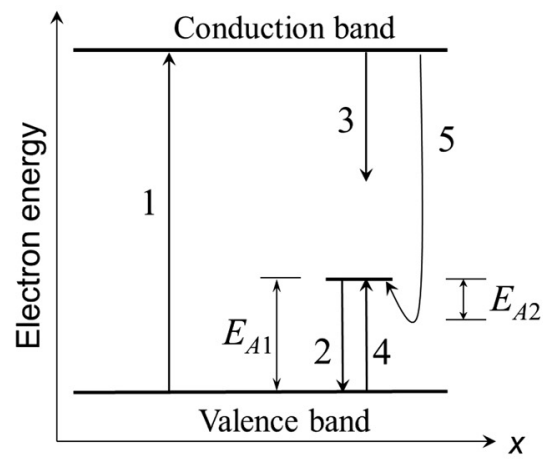

(b)

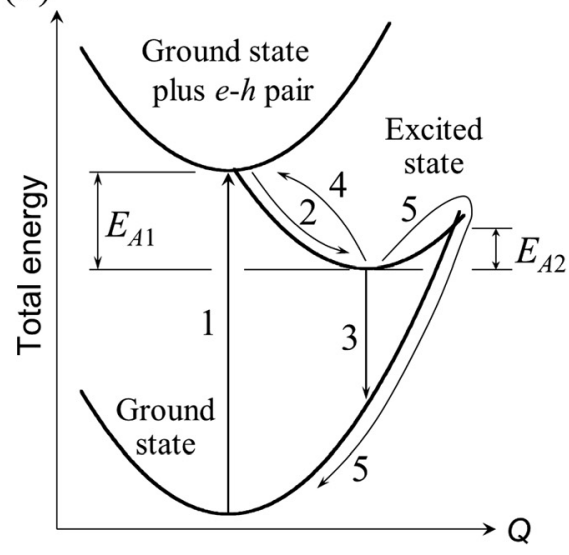

FIG. 1. Schematic energy diagrams for an acceptor in an $n$-type semiconductor. (a) Band diagram. (b) One-dimensional configuration coordinate diagram. The arrows show transitions for electrons. Transition 1 corresponds to an excitation of the electron-hole pair. Transition 2 corresponds to a capture of a free hole by the acceptor and subsequent relaxation of the lattice around it. Transition 3 is a recombination of a free electron with a bound hole resulting in PL. Transition 4 is the thermal emission of holes to the valence band (or, what is the same, the excitation of an electron to the acceptor level). Transition 5 is the thermal excitation of the defect to the point where the adiabatic potentials of the ground and excited states cross and the following nonradiative recombination with emission of large number of phonons. $E_{A 1}$ is the acceptor's ionization energy and $E_{A 2}$ is the barrier height for the nonradiative recombination.

potential minimum of the excited state and the cross-over point of the excited and ground states $\left[E_{A 2}\right.$ in Fig. 1(b)]. The temperature dependence of PL intensity can still be described with Eq. (1). However, in this mechanism,

$$
C \approx \tau_{P L} \omega \text { and } \quad E_{A}=E_{A 2},
$$

where $\omega$ is the vibrational frequency of the defect in the excited state ( $\hbar \omega$ is the energy of the dominant phonon mode of the defect).

In principle, these two mechanisms can be distinguished experimentally when the IQE of a PL band is high enough: in the first mechanism, the quenching of PL from one defect leads to the appearance of extra holes in the valence band and their recombination via other radiative and nonradiative channels. As a result, the quenching of one PL band is accompanied by a rise of intensity in all the other PL bands. ${ }^{1,9}$ In the one-center mechanism, holes do not appear in the valence band during nonradiative quenching of the PL band, and the quenching of this PL band does not affect the intensities of other PL bands. We emphasize that it is easy to distinguish between the two mechanisms only when the IQE of the PL band is close to unity. In the case of the BL band in undoped or Zn-doped GaN, the multi-center mechanism of the PL quenching (caused by the escape of holes to the valence band) was confirmed by the observation of an increase in intensities of the exciton and yellow luminescence bands concurrently with the quenching of the BL band. ${ }^{1,6,10}$

In $p$-type semiconductors, in which the Fermi level is close to the valence band, we also expect to see a temperature dependence of PL that can be described with Eq. (1) and can be explained by one of the two quenching mechanisms. For this case, electrons escape from donor levels to the conduction band and recombine with holes via other channels (multi-center mechanism) or the donor is excited by temperature so that its electron recombines with a free hole nonradiatively after passing through the cross-over point in adiabatic potentials of the donor (one-center mechanism). However, in high-resistivity or insulating samples, where the Fermi level is far from the conduction and valence bands, the PL quenching mechanisms are less understood, and the attribution of the activation energy of the PL quenching should be done with caution.

In this work, we use phenomenological theory to predict possible temperature dependencies of defect-related PL in conductive and high-resistivity semiconductors. These dependencies are compared with available experimental data. Unusual effects, such as abrupt and tunable thermal quenching of PL in semiconductors will be analyzed in more detail. Defects in GaN are of prime interest in this work, yet other III-V and II-VI semiconductor systems will also be reviewed.

\section{PHENOMENOLOGICAL THEORY}

For many semiconductors, and in particular for $\mathrm{GaN}$ and $\mathrm{ZnO}$, a variety of point defects can be reduced to just three major types. The first type is a shallow donor. Shallow donors are often introduced unintentionally during growth and are responsible for $n$-type conductivity. Examples are $\mathrm{O}_{\mathrm{N}}$ or $\mathrm{Si}_{\mathrm{Ga}}$ with the ionization energy, $E_{D}$, of about $30 \mathrm{meV}$ in $\mathrm{GaN}$ and $\mathrm{Ga}_{\mathrm{Zn}}, \mathrm{Al}_{\mathrm{Zn}}$, or $\mathrm{H}$ with $E_{D}=50-60 \mathrm{meV}$ in $\mathrm{ZnO}$. The second type of defects is an acceptor that is responsible for a PL band. Since the electrons bound to the shallow donors have relatively large wave-functions, transitions from shallow donors to different acceptors, the so-called donor-acceptor pair (DAP) recombination, are very efficient, especially at low temperatures when the thermal emission of electrons to the conduction band is negligible. For deep donors, due to high localization of the bound electrons, the DAP transitions have negligible efficiency, unless the concentration of deep donors or acceptors is very high so that the distance between donors and acceptors is small. ${ }^{11}$ At elevated temperatures, the rate of the thermal emission of electrons from shallow donors to the conduction band may become significant. Then, transitions from the conduction band to the acceptor, the so-called $e A$ transitions, may contribute to PL more than the DAP recombination. The third 
type of defects is the dominant center of nonradiative recombination. Inclusion of this type is necessary to explain the less than $100 \%$ quantum efficiency of PL, and sometimes nonradiative defects play an important role in the temperature dependencies of PL as will be shown below. These three types are usually enough to explain different temperature dependencies of the defect-related PL intensity.

Let us consider a direct-bandgap semiconductor with bandgap $E_{g}$, having shallow donors $D$, acceptors $A$ and nonradiative centers $S$ with total concentrations $N_{D}, N_{A}$, and $N_{S}$, respectively (Fig. 2). The ionization energies of donors and acceptors are $E_{D}$ and $E_{A}$, whereas the nonradiative centers $S$ have deep levels, so that carriers cannot escape from them at reasonable temperatures. Later, we will discuss briefly how thermal emission of electrons or holes from the nonradiative centers can affect the temperature dependencies of PL.

Illumination with a laser with above-bandgap energy and excitation power density $P_{\text {exc }}$ creates electron-hole pairs with rate $G\left[\mathrm{~cm}^{-3} \mathrm{~s}^{-1}\right]$. These pairs may form excitons with rate $N_{e x}=C_{e x}^{*} G$ if the electron-hole pairs do not separate upon generation, or with rate $N_{e x}=C_{e x} n p$ if they separate. A competing and often dominant mechanism of electron-hole recombination is recombination via point defects. Transitions via defects occur in two steps: first, one charge carrier (e.g., a hole in an $n$-type semiconductor) must be captured by a defect having an available empty site (the hole may be captured by a negatively charged acceptor or by a neutral donor), then another carrier (a free electron in the above example) is captured by the defect and recombines with the hole. The first or faster transition is usually nonradiative; i.e., the energy is released in the form of lattice oscillations. The second transition may be radiative when a photon is emitted or nonradiative when only phonons are emitted. Usually, small-energy transitions are nonradiative while large-energy transitions are radiative. Recombination

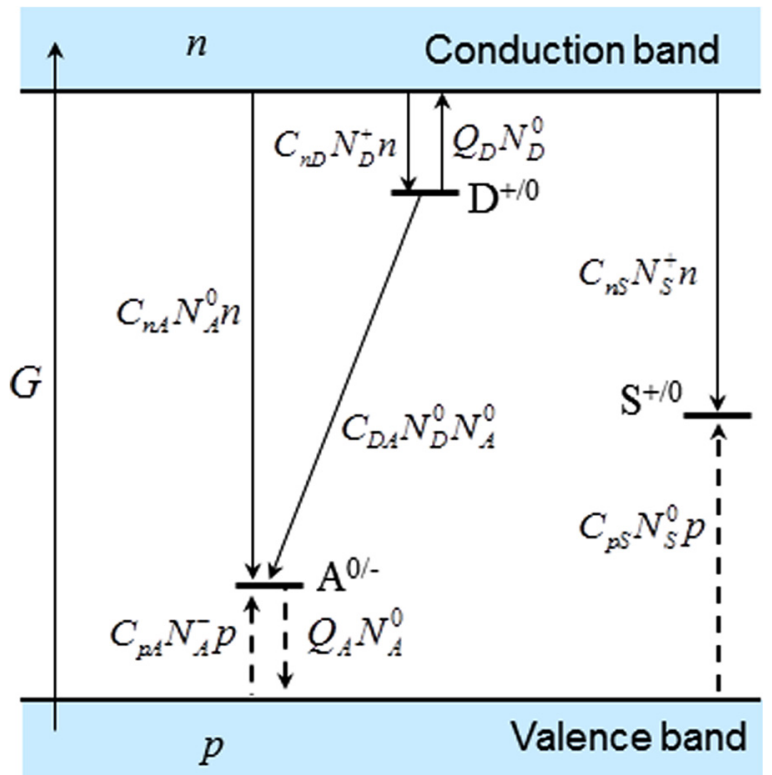

FIG. 2. Energy band diagram and schematic of electron and hole transitions in a semiconductor with three types of defects: a shallow donor $D$, a nonradiative deep donor $S$, and an acceptor $A$. Electron and hole transitions are shown with solid and dashed arrows, respectively. via certain deep-level defects with very large lattice relaxation is always nonradiative. In this case, both transitions cause the emission of multiple phonons. ${ }^{2}$

In wide-bandgap semiconductors with moderate doping levels, there are no free electrons and free holes in the conduction and valence bands, respectively, in dark conditions at very low temperature. Photogenerated electrons are quickly captured by positively charged shallow donors, while holes are mostly captured by negatively charged acceptors. These capture transitions are fast (on the order of $10^{-10} \mathrm{~s}$ ) and nonradiative. Then a tunnel transition of an electron between a spatially separated shallow donor $D$ and acceptor $A$ is possible, with the probability of a transition depending on the sizes of the electron and hole wave functions and the separation between $D$ and $A$. There is a random distribution of the separations, and thus the characteristic time of the transition varies widely for different $D A$ pairs (roughly from nanoseconds to seconds and even years at low temperatures). For simplicity, we will introduce an average probability of such transitions. At low temperatures, the probability of the DAP recombination is much higher than the probability for a photogenerated electron to be captured directly by a neutral acceptor, because the concentration of free electrons is much lower than the concentration of electrons bound to shallow donors. Below, we will consider a model with the transitions indicated by arrows in Fig. 2. Excitonic or band-to-band recombination will be ignored due to its low efficiency relative to nonradiative recombination. However, the model can be easily generalized to account for this recombination channel. ${ }^{12}$

Transition rates are described phenomenologically, in the first approximation, as the product of the concentrations of available carriers and available empty sites, with a constant factor called the capture coefficient. The rate equations under steady-state conditions can be written down from the band diagram shown in Fig. 2 and are given by Eqs. (7)-(12) of Ref. 13. In this figure and the equations, $n$ and $p$ are the concentrations of free electrons and holes, $C_{n D}, C_{n A}$, and $C_{n S}$ are electron capture coefficients for the shallow donor $D$, acceptor $A$, and a nonradiative defect $S$, respectively, $C_{p A}$ and $C_{p S}$ are hole-capture coefficients for the acceptor and nonradiative defect, respectively, and $C_{D A}$ is the effective coefficient for the DAP recombination. For example, the capture rate of free electrons by shallow donors is $C_{n D} n N_{D}^{+}$, where $n$ is the concentration of electrons in the conduction band, $N_{D}^{+}$is the concentration of positively charged donors, and $C_{n D}$ is the electron-capture coefficient for the shallow donor. Coefficients $Q_{D}$ and $Q_{A}$ describe the thermal emission of electrons and holes to the conduction and valence bands, respectively. These coefficients can be found from detailed balance as

$$
Q_{D}=\frac{C_{n D} N_{c}}{g} \exp \left(-\frac{E_{D}}{k T}\right),
$$

and

$$
Q_{A}=\frac{C_{p A} N_{v}}{g} \exp \left(-\frac{E_{A}}{k T}\right)
$$


where $N_{c}$ and $N_{v}$ are the effective density of states in the conduction and valence bands, respectively, and $g$ is the degeneracy factor for the donor and acceptor levels (assumed to be equal to 2 for both). The capture coefficients $C_{n i}$ and $C_{p i}$ are usually related to the capture cross-sections $\sigma_{n i}$ and $\sigma_{p i}$ as

$$
C_{n i}=\sigma_{n i}\left\langle v_{n}\right\rangle=\sigma_{n i} \sqrt{\frac{8 k T}{\pi m_{n}}},
$$

and

$$
C_{p i}=\sigma_{p i}\left\langle v_{p}\right\rangle=\sigma_{p i} \sqrt{\frac{8 k T}{\pi m_{p}}},
$$

where $\left\langle v_{n}\right\rangle$ and $\left\langle v_{p}\right\rangle$ are the mean thermal velocities of electrons in the conduction band and holes in the valence band, respectively, $m_{n}$ and $m_{p}$ are their effective masses, and $i$ labels the donor, acceptor, or nonradiative defect.

Three cases can be distinguished, which will result in qualitatively different temperature dependencies of PL (Fig. 3). These are (i) a conductive $n$-type semiconductor, (ii) a $p$-type semiconductor, and (iii) a semi-insulating ( $n$ - or $p$-type) semiconductor. In a conductive $n$-type semiconductor, $N_{D}>N_{A}$ if the $S$ center is a donor and $N_{D}>N_{A}+N_{S}$ if the $S$ center is an acceptor, with the Fermi level located very close to the shallow donor level. In a $p$-type semiconductor, $N_{A}>N_{D}+N_{S}$ if the $S$ center is a donor and $N_{A}>N_{D}$ if the $S$ center is an acceptor, with the Fermi level located close to the acceptor level. Finally, in a semi-insulating semiconductor, $N_{D}+N_{S}>N_{A}>N_{D}$ (formally $n$-type) if the $S$ center is a donor and $N_{A}+N_{S}>N_{D}>N_{A}$ (formally $p$-type) if the $S$ center is an acceptor, with the Fermi level located close to the deep level of the $S$ center. Our calculations show that the temperature dependencies for a conductive $n$-type semiconductor are qualitatively the same when the $S$ center is a donor or an acceptor. The same can be said about insulating semiconductors. However, we noticed significant differences in the dependencies for $p$-type semiconductors between the cases when the $S$ center is a donor or an acceptor. Below, the three distinctive cases will be considered in more detail.

\section{A. Conductive $n$-type semiconductor}

When the Fermi level in dark is close to the conduction band (for a conductive $n$-type semiconductor), all acceptors and nonradiative defects are filled with electrons, while shallow donors are partially populated with electrons. At low enough excitation intensity (when the PL intensity increases linearly with excitation intensity), the concentration of holes at the acceptor level is negligible; i.e., $N_{A}^{0} \ll N_{A}$, and a simple analytical expression can be obtained for the quantum efficiency of PL caused by transitions from the conduction band and the shallow donor to an acceptor: ${ }^{1}$

$$
\eta(T)=\frac{I^{D A P}+I^{e A}}{G}=\frac{\eta_{0}}{1+\left(1-\eta_{0}\right) \tau_{P L} C_{p A} \frac{N_{v}}{g} \exp \left(-\frac{E_{A}}{k T}\right)},
$$

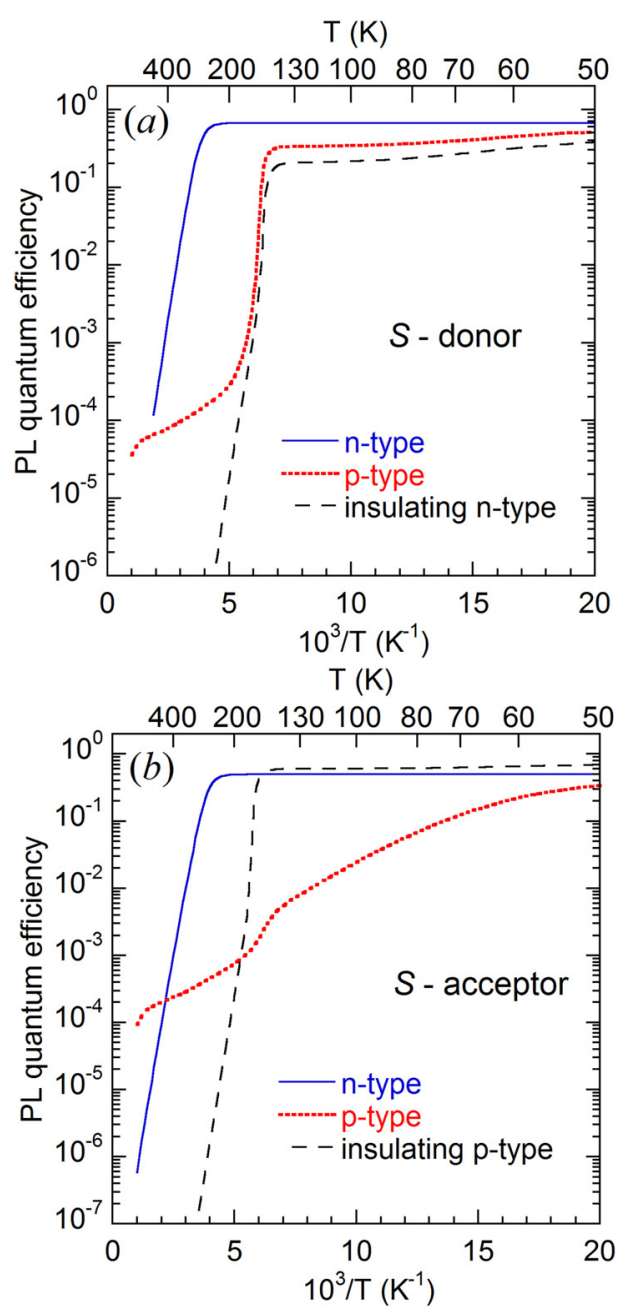

FIG. 3. Temperature dependence of the PL quantum efficiency for a system of three defects when the $S$ center is a deep donor (a) or an acceptor (b). The following parameters are used in the calculations: (a) $N_{S}=1 \times 10^{18} \mathrm{~cm}^{-3}\left(n-\right.$ and $p$-type) and $2 \times 10^{18} \mathrm{~cm}^{-3}$ (insulating), $N_{D}=2.5 \times 10^{18} \mathrm{~cm}^{-3}$ ( $n$-type) and $0.5 \times 10^{18} \mathrm{~cm}^{-3}$ ( $p$-type and insulating), $N_{A}=2 \times 10^{18} \mathrm{~cm}^{-3} \quad$ (all three cases); (b) $N_{S}=1 \times 10^{18} \mathrm{~cm}^{-3}$ (all three cases), $N_{D}=2.5 \times 10^{18} \mathrm{~cm}^{-3}$ (n-type and insulating) and $0.5 \times 10^{18} \mathrm{~cm}^{-3}$ ( $p$-type), $N_{A}=1 \times 10^{18} \mathrm{~cm}^{-3}$ ( $n$-type) and $2 \times 10^{18} \mathrm{~cm}^{-3}$ ( $p$-type and insulating). Other parameters are the same for all the cases: $C_{n S}=C_{n D}=10^{-8}$ $\mathrm{cm}^{3} / \mathrm{s}, C_{p S}=C_{p A}=10^{-6} \mathrm{~cm}^{3} / \mathrm{s}, C_{n A}=C_{D A}=10^{-12} \mathrm{~cm}^{3} / \mathrm{s}, E_{D}=30 \mathrm{meV}$, $E_{A}=350 \mathrm{meV}$, and $G=10^{20} \mathrm{~cm}^{-3} \mathrm{~s}^{-1}$.

where $\tau_{P L}=\left(C_{n A} n\right)^{-1}$ is the PL lifetime, and $\eta_{0}$ is the IQE of this PL at low temperatures, before quenching, which is given by the expression ${ }^{13}$

$$
\eta_{0} \approx \frac{C_{p A} N_{A}}{C_{p A} N_{A}+C_{p S} N_{S}}
$$

The quenching of the acceptor-related PL band starts when the second term in the denominator of Eq. (8) becomes equal to 1 ; i.e., at

$$
T_{0}=\frac{E_{A}}{k \ln \left[\left(1-\eta_{0}\right) \tau_{P L} C_{p A} g^{-1} N_{v}\right]} .
$$

The activation energy of this quenching is close to $E_{A}$ (it is slightly larger due to the temperature dependence of $N_{v}$ ). For the examples shown in Fig. 3, the PL quenching begins at $T_{0} \approx 270 \mathrm{~K}$, and the slope of the quenching in the Arrhenius 
plot corresponds to $E_{A}=350 \mathrm{meV}$. Note that, according to Eq. (10), $T_{0}$ is independent of the excitation intensity (provided that the acceptor is not saturated with holes which may happen at high excitation intensity).

The quenching of the PL related to a defect of type $i=1$ results in a coherent rise of the PL intensity for all recombination channels with $i=2,3, \ldots$. The relative change of the PL intensity for these channels, $I_{i}^{P L} / I_{i 0}^{P L}$ due to the quenching of the PL band with $i=1$ can be expressed as ${ }^{10,12}$

$$
\frac{I_{i}^{P L}}{I_{i 0}^{P L}}=\frac{1-\eta_{1}(T)}{1-\eta_{10}(T)}
$$

where $\eta_{1}$ is the IQE of the PL via the defect with $i=1$ and $\eta_{10}$ is the IQE of this PL at low temperatures, before its quenching.

\section{B. Conductive or high-resistivity p-type semiconductor}

\section{The $S$ center is a donor}

Let us consider a $p$-type semiconductor with the Fermi level located close to an acceptor level in dark. We will assume that $E_{A} \gg E_{D}$, as in the case of GaN or $\mathrm{ZnO}$. The sample is conductive if the acceptor level is close to the valence band, and it is high-resistivity if the acceptor level is deep. It is likely that the dominant nonradiative center is a donor, because the formation of donors is facilitated in $p$-type semiconductors due to the self-compensation effect. ${ }^{14}$ In this case, $N_{A}>N_{D}+N_{S}$, and both shallow donors and nonradiative defects are empty in dark. The numerical solution of the rate equations shows that in conditions of continuous illumination with above-bandgap light at low temperature, shallow donors are mostly empty $\left(N_{D}^{+} \approx N_{D}\right)$, while nonradiative defects become partially filled with electrons even at very low intensity of light. ${ }^{13}$

At very low temperatures $(T<20 \mathrm{~K}$ for $\mathrm{GaN})$, when the thermal emission of electrons from the shallow donors to the conduction band can be ignored, the population of the $S$ centers with electrons depends on the capture coefficients for competing recombination channels:

$$
N_{S}^{+}=\frac{N_{D}}{2}\left[\sqrt{(1+\delta)^{2}+4 \delta \frac{N_{S}}{N_{D}}}-(1+\delta)\right],
$$

where $\delta=\left(C_{n D} C_{p S}\right) /\left(C_{n S} C_{p A}\right)$. Note that $N_{S}^{+} \approx \delta N_{S}$ when $\delta \ll 1$, and $N_{S}^{+} \approx N_{S}$ when $\delta \gg 1$. Figure 4(a) shows how the population of the $S$ centers with electrons depends on the parameter $\delta$. In the limit of low temperatures, only DAPtype transitions contribute to the acceptor-related PL, and the $\mathrm{PL} \mathrm{IQE} \mathrm{is}$

$$
\eta=\frac{1}{1+\frac{C_{p S}\left(N_{S}-N_{S}^{+}\right)}{C_{p A}\left(N_{D}+N_{S}^{+}\right)}}
$$

where $N_{S}^{+}$is given by Eq. (12).
With increasing temperature (from 30 to $70 \mathrm{~K}$ for $\mathrm{GaN}$ ), electrons start escaping from the shallow donor to the conduction band. This leads to a gradual increase of $n, N_{D}^{0}$, and the intensity of the $e A$ luminescence, all with the activation energy of $E_{D}$. Simultaneously, $N_{S}^{+}$decreases, so that the $S$ centers become almost completely filled with electrons $\left(N_{S}^{0} \approx N_{S}\right)$ due to the increased concentration of electrons in the conduction band. Figure 4(b) shows an example for the population of the $S$ center with electrons. Typically, the nonradiative capture coefficient $C_{n D}$ is larger than the radiative capture coefficient $C_{n A}$ by several orders of magnitude. Then, we expect that $N_{S}^{0} \approx N_{S}$ for $\delta<1$. Since the capture coefficients $C_{n A}$, and $C_{p A}$ can be found from PL experiments on conductive $n$-type material containing same acceptors, ${ }^{1,12}$ the restriction on $\delta$ required to saturate the $S$ center with electrons at these temperatures gives an estimate for the $C_{n S} / C_{p S}$ ratio. Thus, by studying temperature dependencies of PL, certain properties of dominant nonradiative defects can be determined.

The IQE of the acceptor-related PL ( $e A$ plus DAP) at these temperatures $[70-130 \mathrm{~K}$ in Fig. 3(a)] is equal to

$$
\eta \approx \eta_{0}=\frac{1}{1+\frac{C_{p S} N_{S}}{C_{p A} N_{D}}} .
$$
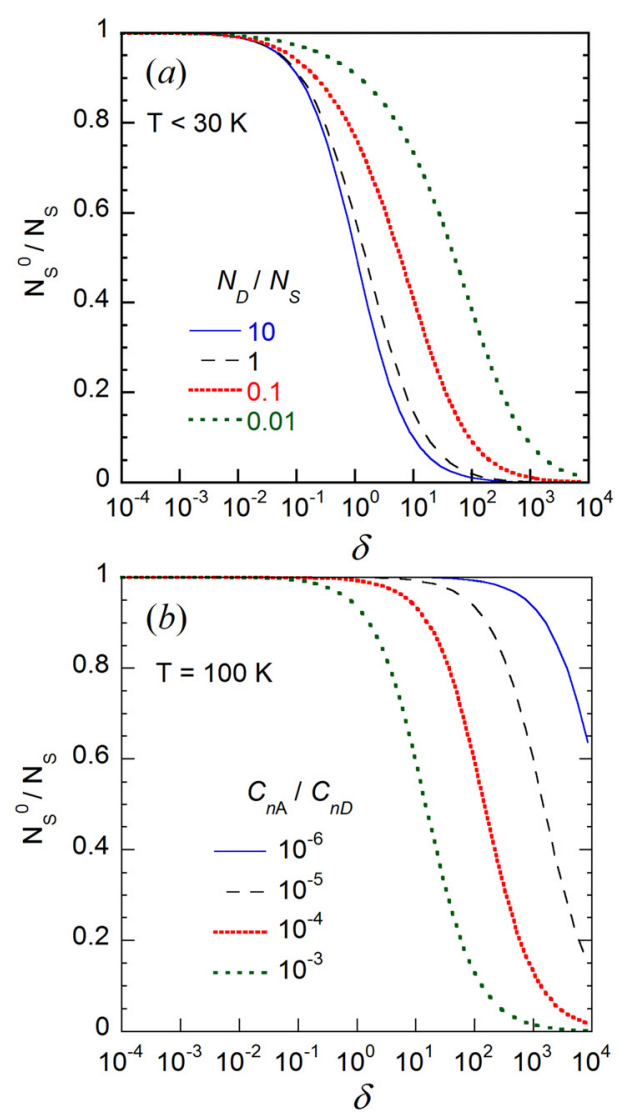

FIG. 4. Dependence of the population of the $S$ centers with electrons on parameter $\delta$. (a) Region of very low temperatures $(T<30 \mathrm{~K}$ for $\mathrm{GaN})$. The $N_{D} / N_{S}$ ratio is varied. (b) Intermediate temperatures $(T \approx 100 \mathrm{~K}$ for $\mathrm{GaN})$. $N_{D}=10^{17} \mathrm{~cm}^{-3}, N_{A}=10^{18} \mathrm{~cm}^{-3}, E_{D}=30 \mathrm{meV}, C_{D A}=C_{n A}$. 
In this temperature region, an inversion of conductivity type should be observed, because $n \gg p$. At temperatures lower but close to $T_{0}$,

$$
\frac{n}{p}=\frac{C_{p A} N_{D}}{C_{n A}\left(N_{A}-N_{D}\right)} \approx \frac{C_{p A}}{C_{n A}} .
$$

The coefficient $C_{p A}$ is large for the nonradiative capture of holes, especially for acceptors that attract holes by their negative charge. The coefficient $C_{n A}$ for the radiative recombination is typically smaller by several orders of magnitude. Note that, although the $n \gg p$ condition is expected to be a typical case for $p$-type semiconductors with $E_{A} \gg E_{D}$, the abrupt quenching of PL will not be observed for all such samples. For this to happen, certain properties of the dominant nonradiative center are needed.

At higher temperatures $[T>150 \mathrm{~K}$ for $p$-type in Fig. 3(a)], the thermal emission of holes from the acceptor level to the valence band becomes significant. The contribution of the emitted holes in the valence band increases exponentially with temperature, proportionally to $\exp \left(-E_{A} / k T\right)$. In a narrow temperature range, the population of the $S$ centers with electrons may change dramatically, from $N_{S}^{0} \approx N_{S}$ to $N_{S}^{0} \ll N_{S}$. As it is explained in Ref. 13, the thermally emitted holes change the balance in the system, and the system undergoes a transition from a state with the population inversion (the deep donors are saturated with electrons and the acceptors accumulate holes) to a quasi-equilibrium state in a narrow range of temperatures. Simultaneously, the concentration of free electrons and the PL intensity abruptly drop. The critical temperature is

$$
T_{0} \approx \frac{E_{A}}{k \ln (B / G)},
$$

with

$$
B=\frac{C_{p S} N_{S}\left(N_{A}-N_{D}\right) N_{v}}{g N_{D}} .
$$

Thus, with increasing generation rate, $T_{0}$ increases logarithmically. This tunable behavior is well seen in Fig. 5(a), where the generation rate is varied by six orders of magnitude.

The value of the drop, $R$, in PL intensity at $T \approx T_{0}$ is

$$
R=\frac{\eta_{0} C_{n S} N_{S}}{\left[C_{n A}+C_{D A} \frac{g N_{D}}{N_{c}} \exp \left(\frac{E_{D}}{k T_{0}}\right)\right]\left(N_{A}-N_{S}-N_{D}\right)}
$$

where $\eta_{0}$ is defined in Eq. (14). In the example shown in Fig. 3(a) for a $p$-type semiconductor, $\eta_{0}=0.33, T_{0}=160 \mathrm{~K}$, and $R=700$. For dependencies shown in Fig. 5(a), $T_{0}$ increases from 137 to $243 \mathrm{~K}$, and $R$ changes from 400 to 2000 as $G$ increases from $10^{18}$ to $10^{24} \mathrm{~cm}^{-3} \mathrm{~s}^{-1}$.

Questions can be raised as to how common is the abrupt tunable quenching in $p$-type semiconductors, and which parameters of the model favor the observation of this phenomenon? In Figs. 6-9, temperature dependencies for $\mathrm{PL}$ in a $p$-type semiconductor are shown where parameters of the model are changed one by one. Figure 6 shows the
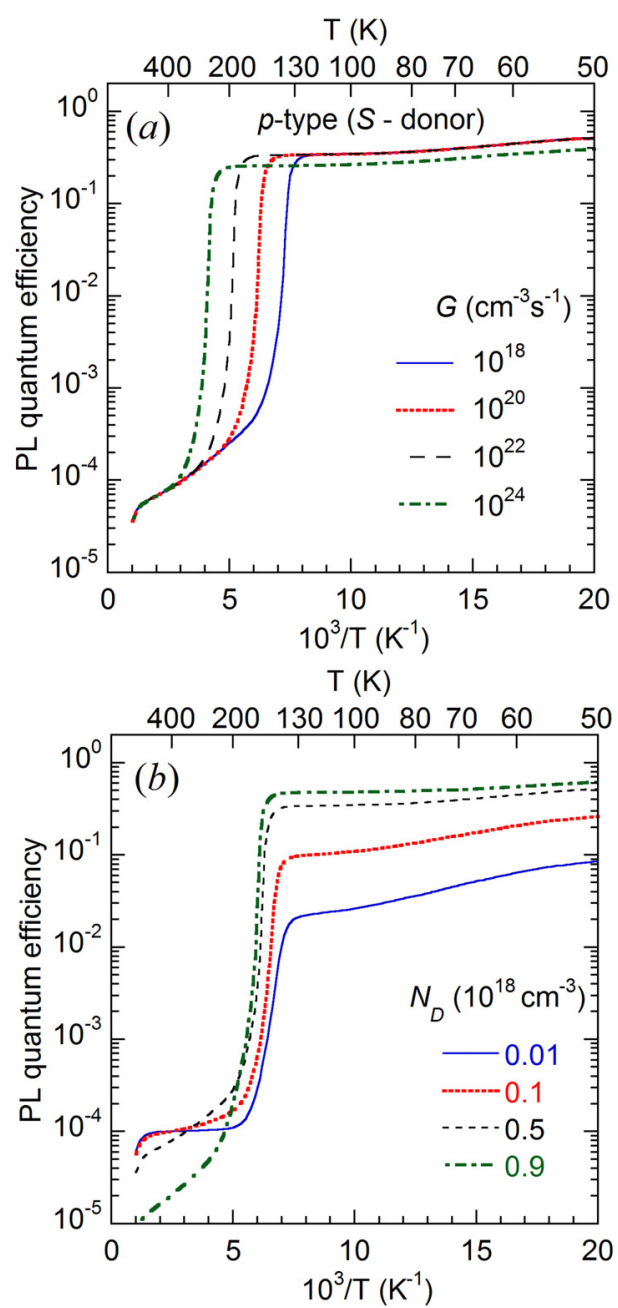

FIG. 5. Temperature dependencies of the PL quantum efficiency for a $p$-type semiconductor with the $S$ center being a deep donor. Model parameters: $N_{S}=1 \times 10^{18} \mathrm{~cm}^{-3}, N_{D}=0.5 \times 10^{18} \mathrm{~cm}^{-3}, N_{A}=2 \times 10^{18} \mathrm{~cm}^{-3} ; C_{n S}=C_{n D}$ $=10^{-8} \mathrm{~cm}^{3} / \mathrm{s}, C_{p S}=C_{p A}=10^{-6} \mathrm{~cm}^{3} / \mathrm{s}, \quad C_{n A}=C_{D A}=10^{-12} \mathrm{~cm}^{3} / \mathrm{s}, E_{D}=30$ $\mathrm{meV}, E_{A}=350 \mathrm{meV}, G=10^{20} \mathrm{~cm}^{-3} \mathrm{~s}^{-1}$. (a) Parameter $G$ is varied. (b) Parameter $N_{D}$ is varied as indicated.

temperature dependencies where the electron-capture coefficients for the $S$ center and for the shallow donor are varied between $10^{-12}$ and $10^{-6} \mathrm{~cm}^{3} / \mathrm{s}$. We can see in Fig. 6(a) that it is crucial to have a large electron-capture coefficient for the $S$ center in order to observe a significant and abrupt drop in the PL intensity. At $T<T_{0}$, the PL intensity is not very sensitive to $C_{n S}$, but at $T>T_{0}$, the PL IQE is inversely proportional to $C_{n S}$. At temperatures above $T_{0}$, electrons are minority carriers in a $p$-type semiconductor. Then, competition for the capture of free electrons between the $S$ center and the $A$ center determines the PL IQE. The higher the $C_{n A} / C_{n S}$ ratio (or $C_{D A} / C_{n S}$ if the DAP recombination dominates over the $e A$ recombination), the higher is the PL efficiency at $T>T_{0}$.

In contrast, by varying parameter $C_{n D}$ in wide range, we do not observe any detectable changes in position and magnitude of the PL drop at $T \approx T_{0}$ [Fig. 6(b)]. The most significant change can be noticed at very low temperatures $(T<50 \mathrm{~K})$, where the PL IQE increases with increasing $C_{n D}$. It approaches unity when $C_{n D} \gg C_{n S}$ (or at $\delta \gg 1$ ), because electrons are more efficiently captured by the shallow donors than by the nonradiative defects. 

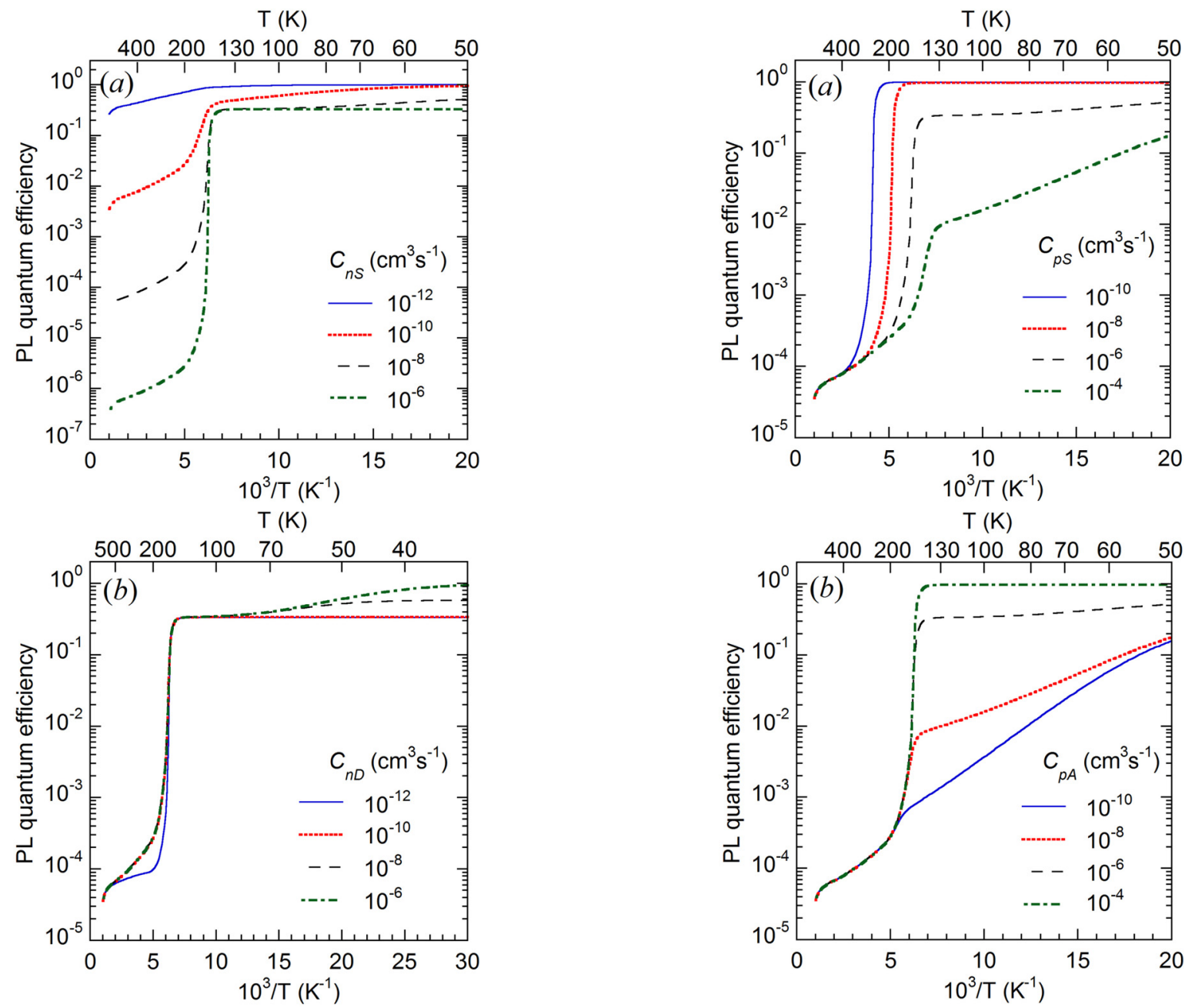

FIG. 6. Temperature dependence of the quantum efficiency of PL for $p$-type ( $S$ - donor). (a) $C_{n S}$ is varied. (b) $C_{n D}$ is varied. Other parameters are the same as in Fig. 5.

Figure 7 shows the temperature dependencies for $\eta$ where the hole-capture coefficients for the $S$ center and for the acceptor are varied between $10^{-10}$ and $10^{-4} \mathrm{~cm}^{3} / \mathrm{s}$. By increasing the $C_{p A} / C_{p S}$ ratio, the PL IQE at $T<T_{0}$ increases and approaches unity when $C_{p A} \gg C_{p S}$. In agreement with Eq. (14), the parameters $C_{n D}, C_{n S}, C_{n A}$, and $C_{D A}$ do not affect the low-temperature PL IQE. Thus, in the temperature range preceding the abrupt thermal quenching, the competition for holes between different recombination channels determines the PL efficiency. In the extreme case, when $C_{p A}$ is smaller than $C_{p S}$ by several orders of magnitude, the PL IQE decreases with temperature as

$$
\eta \approx \frac{\left[C_{n A}+C_{D A} \frac{N_{D} g}{N_{c}} \exp \left(\frac{E_{D}}{k T}\right)\right]\left(N_{A}-N_{S}-N_{D}\right)}{C_{n S} N_{S}},
$$

and no drop at $T \approx T_{0}$ is observed. The position of the abrupt drop is independent of $C_{p A}$ [Fig. 7(b)], yet it shifts to higher temperatures with decreasing $C_{p S}$ [Fig. 7(a)]. The value of the drop $R$ decreases as the $C_{p A} / C_{p S}$ ratio decreases and it vanishes when $C_{p A} / C_{p S} \rightarrow 0$. Note that the value of $C_{p A}$ can be reliably determined from the temperature dependence of

FIG. 7. Temperature dependence of the quantum efficiency of PL for $p$-type ( $S$ - donor). (a) $C_{p S}$ is varied. (b) $C_{p A}$ is varied. Other parameters are the same as in Fig. 5.

PL in conductive $n$-type samples when the PL is related to the same acceptor. ${ }^{1,6}$

Figure 8 shows the temperature dependencies where the electron-capture coefficients for the acceptor are varied between $10^{-14}$ and $10^{-8} \mathrm{~cm}^{3} / \mathrm{s}$. This is a reasonable range for the radiative transitions responsible for the $e A$ and DAP recombination. We can see that varying these parameters affects the value of the drop $R$ and changes the shape of the temperature dependence at $T>T_{0}$. This behavior agrees with Eq. (18), and $R$ decreases accordingly as both $C_{n A}$ and $C_{D A}$ increase. Note that these parameters characterize the PL lifetime in $n$-type materials and therefore can be estimated for a particular type of acceptor. ${ }^{6}$

Finally, the effect of the ionization energies on the temperature dependencies of PL is shown in Fig. 9. With increasing $E_{D}$, the drop in PL intensity becomes smaller and less abrupt. However, shallow donors with small ionization energy (about 30 and $50 \mathrm{meV}$ in $\mathrm{GaN}$ and $\mathrm{ZnO}$, respectively) are always present in many semiconductors, and they will contribute the most to the temperature dependence of defectrelated PL. As for the acceptors, in agreement with Eq. (16), the abrupt quenching shifts to higher temperatures for deeper defects and $T_{0} \propto E_{A}$. Also, the value of the drop and its 
$\mathrm{T}(\mathrm{K})$

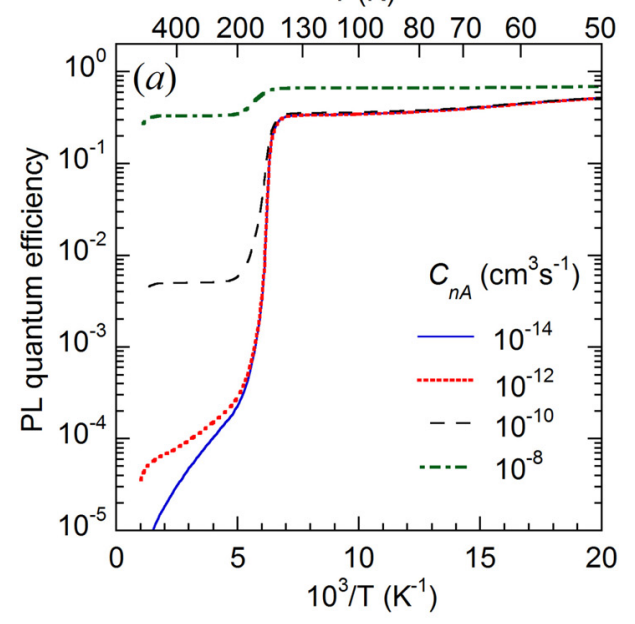

$\mathrm{T}(\mathrm{K})$

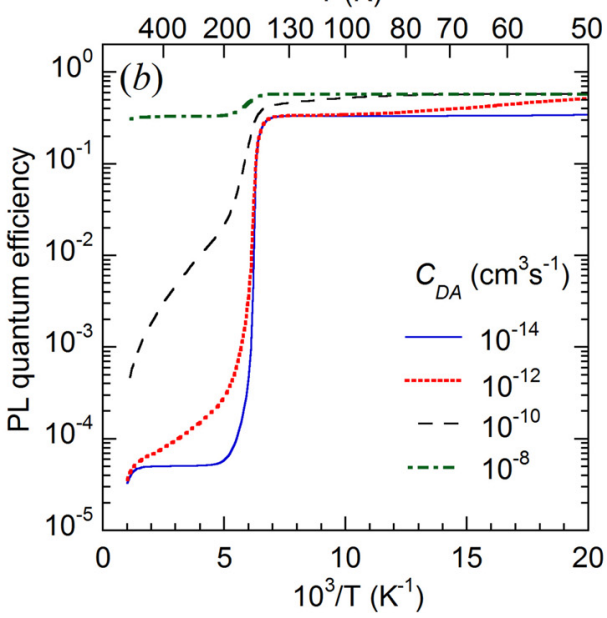

FIG. 8. Temperature dependence of the quantum efficiency of PL for $p$-type ( $S$ - donor). (a) $C_{n A}$ is varied. (b) $C_{D A}$ is varied. Other parameters are the same as in Fig. 5.

abruptness increase with increasing acceptor ionization energy.

\section{The $S$ center is an acceptor}

In a conductive $n$-type semiconductor, it is likely that the dominant nonradiative center is an acceptor and is formed due to the self-compensation effect. Although in $p$ type semiconductors formation of acceptors is unfavorable, we will consider for generality a case when the $S$ center is a deep acceptor. Temperature dependencies for three types of semiconductors for this case are shown in Fig. 3(b). We can see from the comparison of Figs. 3(a) and 3(b) that, for $n$ type semiconductors, the temperature behavior of PL is the same when the $S$ center is a donor or an acceptor. However, for $p$-type semiconductors, especially when the Fermi level is stabilized by an acceptor level $\left(N_{A}>N_{D}\right)$, the temperature dependencies look different. The dependencies for the case of $S$ center being a deep acceptor in a $p$-type semiconductor are shown in Fig. 10(a) for several excitation intensities. The drop in PL efficiency which can be tuned by excitation intensity is observed. But, in contrast to the case when the $S$ center is a donor, the drop is very small. This happens when
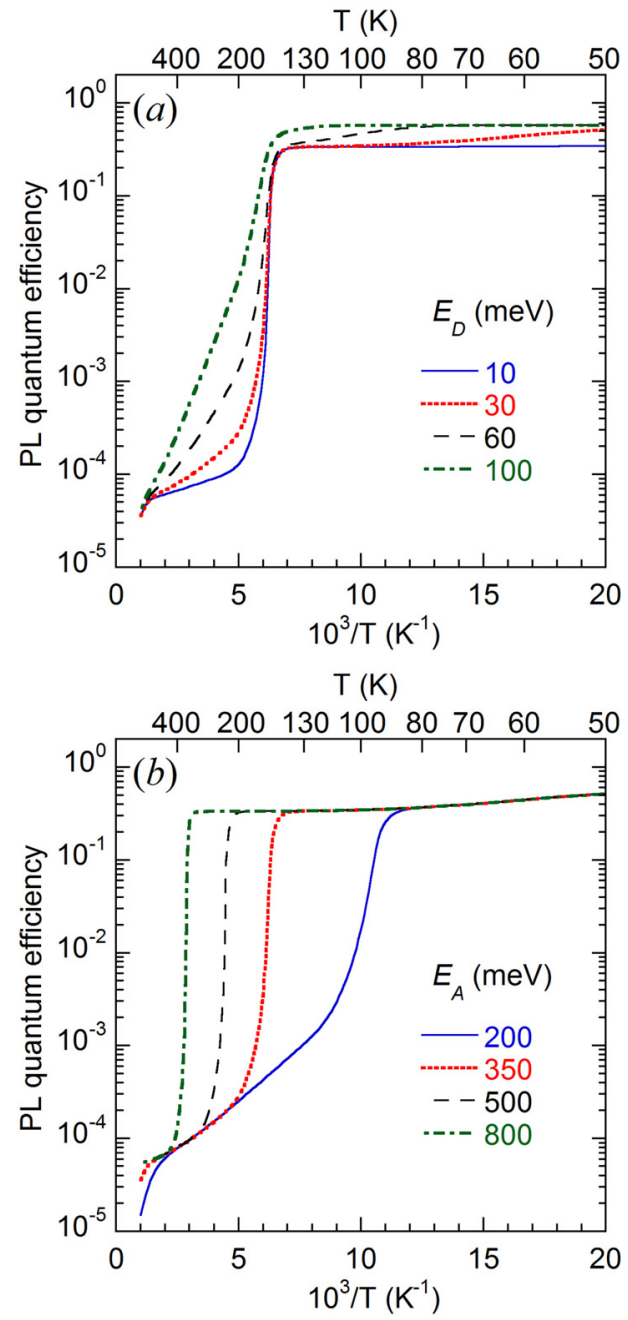

FIG. 9. Temperature dependence of the quantum efficiency of PL for $p$-type ( $S$ - donor). (a) $E_{D}$ is varied. (b) $E_{A}$ is varied. Other parameters are the same as in Fig. 5.

$N_{S}>N_{D}$. Then, at temperatures below the PL drop $(20-100 \mathrm{~K}), N_{A}^{-} \ll N_{A}$ and $N_{S}^{-} \approx N_{D}$. The PL IQE is

$$
\eta \approx \frac{N_{A}\left[C_{n A}+C_{D A} \frac{N_{D} g}{N_{c}} \exp \left(\frac{E_{D}}{k T}\right)\right]}{C_{n S}\left(N_{S}-N_{D}\right)} .
$$

The exponential decrease of PL intensity with activation energy $E_{D}$ is due to the dominance of the second term in the square brackets. The value of the PL intensity drop at $T \approx T_{0}$ is

$$
R \approx \frac{N_{A} N_{S}}{\left(N_{S}-N_{D}\right)\left(N_{A}-N_{D}\right)} .
$$

It can be seen from Eq. (21) that for very low $N_{D}$, the drop is negligible $\left(R \approx 1\right.$ when $\left.N_{D} \ll N_{A}, N_{S}\right)$. The value of the drop increases with increasing $N_{D}$. However, Eq. (21) is not valid when $N_{D}$ is very close to $N_{S}$ or exceeds it.

The temperature dependencies of the PL IQE for different $N_{D}$ are shown in Fig. 10(b). We can see that the abrupt quenching is obvious when $N_{D}>N_{S}$. Then, at temperatures 

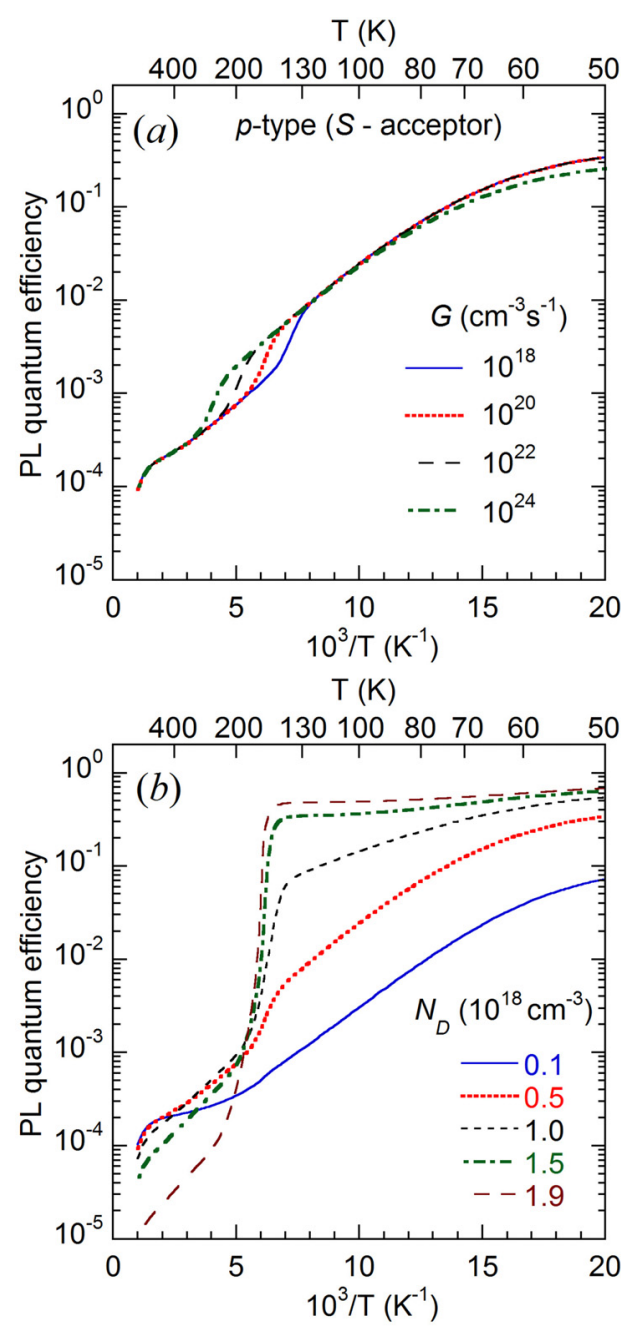

FIG. 10. Temperature dependencies of the PL quantum efficiency for $p$-type with the $S$ center being a deep acceptor. Model parameters: $N_{S}=1 \times 10^{18} \mathrm{~cm}^{-3}, N_{D}=0.5 \times 10^{18} \mathrm{~cm}^{-3}, N_{A}=2 \times 10^{18} \mathrm{~cm}^{-3} ; C_{n S}=C_{n D}$ $=10^{-8} \mathrm{~cm}^{3} / \mathrm{s}, C_{p S}=C_{p A}=10^{-6} \mathrm{~cm}^{3} / \mathrm{s}, C_{n A}=C_{D A}=10^{-12} \mathrm{~cm}^{3} / \mathrm{s}, E_{D}=30$ $\mathrm{meV}, E_{A}=350 \mathrm{meV}$, and $G=10^{20} \mathrm{~cm}^{-3} \mathrm{~s}^{-1}$. (a) Parameter $G$ is varied. (b) Parameter $N_{D}$ is varied as indicated.

below the PL drop $(50-100 \mathrm{~K}), \quad N_{S}^{0} \ll N_{S}$ and $N_{A}^{-}$ $\approx N_{D}-N_{S}$. The PL IQE is

$$
\eta \approx \frac{C_{p A}\left(N_{D}-N_{S}\right)}{C_{p S} N_{S}+C_{p A}\left(N_{D}-N_{S}\right)} .
$$

The value of the drop at $T \approx T_{0}$ is

$$
R \approx \frac{C_{p A} N_{S}}{\left(N_{A}-N_{D}\right)\left(\frac{C_{p S} N_{S}}{C_{n S}\left(N_{D}-N_{S}\right)}+1\right)\left(C_{n A}+C_{D A} \frac{N_{D} g}{N_{c}} \mathrm{e}^{E_{D} / k T}\right)} .
$$

Note that when $N_{D}$ exceeds $N_{A}$, the semiconductor becomes insulating, and the Fermi level in dark is located near the $S$ center.

\section{Insulating semiconductors}

In this paper, we call semiconductors insulating when the Fermi level in dark is close to the deep level of the $S$ center, from which thermal emission of carriers is negligible at temperatures achieved in the experiments. Two cases can be considered: when the $S$ center is a donor (then $N_{D}+N_{S}>N_{A}>N_{D}$ ) and when it is an acceptor (then $N_{A}+N_{S}>N_{D}>N_{A}$ ). In both cases, an abrupt thermal quenching is often observed at $T \approx T_{0}$. The only difference between the temperature dependencies of PL in $p$-type semiconductors and in the insulating samples is that after the drop at $T \approx T_{0}$, the PL intensity decreases with activation energy $E_{D}$ (or completely saturates) in $p$-type samples (Figs. 5 and 10) and it decreases with activation energy $E_{A}$ in insulating samples (Fig. 11). Physically, in p-type samples acceptors are partially or completely filled with holes at $T>T_{0}$, while in insulating samples, acceptors are filled with electrons at $T>T_{0}$. On the other hand, for both types of samples, a population inversion is observed at $T<T_{0}$ (when the $S$ center is almost completely filled with electrons, and the acceptor is filled with holes), and a quasi-equilibrium population is observed at $T>T_{0}$ (when the $S$ center is empty or partially filled with electrons, and the acceptor is partially
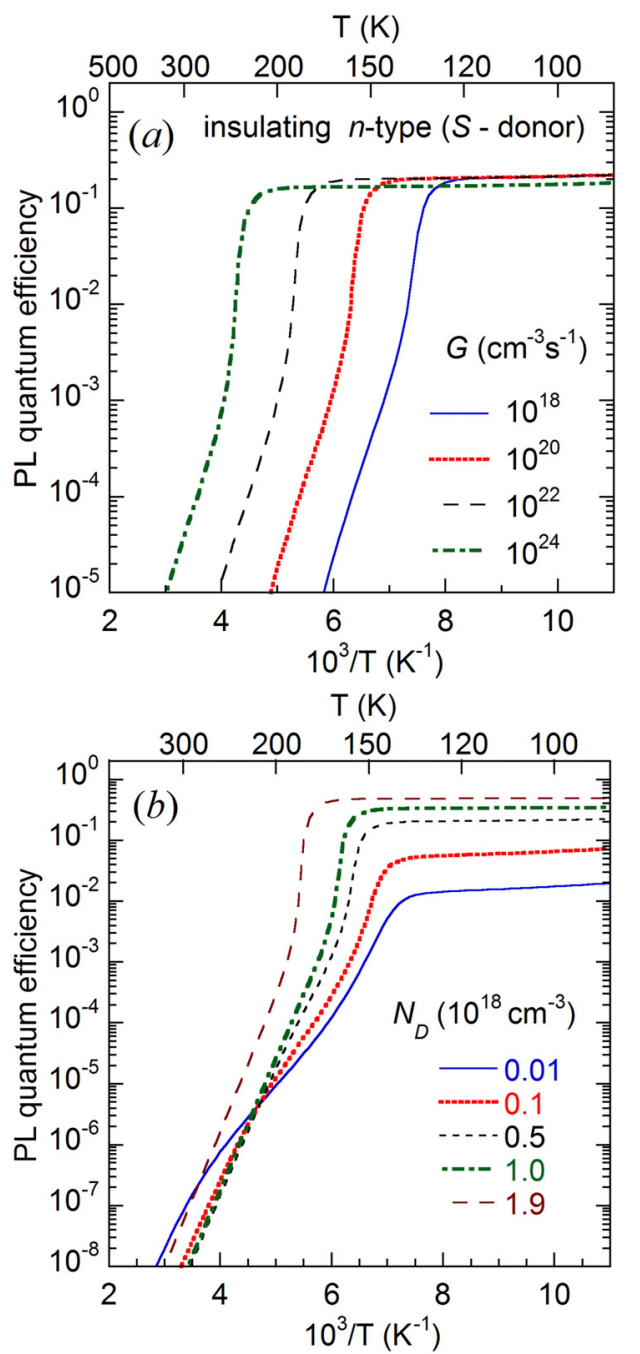

FIG. 11. Temperature dependencies of the PL quantum efficiency for an insulating semiconductor with the $S$ center being a deep donor. Model parameters: $N_{S}=2 \times 10^{18} \mathrm{~cm}^{-3}, N_{D}=0.5 \times 10^{18} \mathrm{~cm}^{-3}, N_{A}=2 \times 10^{18} \mathrm{~cm}^{-3}$; $C_{n S}=C_{n D}=10^{-8} \mathrm{~cm}^{3} / \mathrm{s}, C_{p S}=C_{p A}=10^{-6} \mathrm{~cm}^{3} / \mathrm{s}, C_{n A}=C_{D A}=10^{-12} \mathrm{~cm}^{3} / \mathrm{s}$, $E_{D}=30 \mathrm{meV}, E_{A}=350 \mathrm{meV}$, and $G=10^{20} \mathrm{~cm}^{-3} \mathrm{~s}^{-1}$. (a) Parameter $G$ is varied. (b) Parameter $N_{D}$ is varied as indicated. 
or completely filled with holes, depending on whether the $S$ center is a donor or an acceptor).

\section{DISCUSSION}

\section{A. Historical overview}

Originally, the thermal quenching of PL from defects in solids was explained by the assumption that a defect undergoes a transition from a radiative state to a nonradiative state (the one-center model). This mechanism was first suggested by Seitz ${ }^{7}$ and Gurney and Mott ${ }^{8}$ in 1939 and it is often called the Seitz-Mott mechanism. The essence of the mechanism is that with increasing temperature, a defect may overcome a potential barrier separating the excited and ground states and release energy in the form of multiple phonons instead of emitting a photon [Fig. 1(b)]. If the excited state represents a neutral acceptor that binds a hole and a free electron in the conduction band of an $n$-type semiconductor, the recombination by the Seitz-Mott mechanism would result in a nonradiative recombination between the free electron and bound hole with the emission of a large number of phonons (transition 5 in Fig. 1).

An alternative model that explains thermal quenching of PL in semiconductors was formulated by Schön ${ }^{4}$ in 1942 and further developed by Klasens, ${ }^{5}$ and therefore is called the Schön-Klasens mechanism. We also call this mechanism the multi-center model because the explanation requires the presence of several types of defects in a solid. According to this mechanism, the defect remains radiative at all temperatures; however, the competition between different types of defects causes changes in PL intensity. For example, the thermal emission of holes from an acceptor to the valence band in an $n$-type semiconductor results in the re-capture of these holes by other types of defects. This would lead to the reduction of PL intensity related to the acceptor and to a rise of the recombination rate via all other recombination channels.

In both models, the temperature dependence of the PL efficiency can be described with Eq. (1). However, the meaning of parameters $C$ and $E_{A}$ is different for the two models. While in the Seitz-Mott mechanism $C$ and $E_{A}$ describe the probability and the barrier height for a transition between adiabatic potentials of the same defect, in the Schön-Klasens mechanism these parameters describe the free-carrier capture cross-section and the defect's ionization energy, respectively. Unfortunately, it is usually difficult to distinguish between the two mechanisms because the parameters in these models are often similar, and the secondary evidence (such as the rise of intensities of other PL bands in the Schön-Klasens mechanism) is often negligible and remains unnoticed.

From our experience, the Schön-Klasens model is by far the dominant mechanism of the thermal quenching of defectrelated PL in semiconductors, at least in GaAs, GaN, and $\mathrm{ZnO}$. However, the Seitz-Mott model is better known and more frequently used. Among the most important works on the Seitz-Mott model are papers by Williams, Johnson, and Eyring, ${ }^{15}$ Klick,${ }^{16}$ de la Garanderie and Curie, ${ }^{17}$ Hwang, ${ }^{18}$ and others, who explained the thermal quenching of PL from deeplevel defects by using a one-dimensional configuration coordinate diagram. The configuration coordinate model (often used by photo-chemists) successfully explained optical properties of insulators such as alkali halides. ${ }^{2,19}$ Interestingly, while for many defects in semiconductors there is no clear proof for the Seitz-Mott mechanism of thermal quenching, this model commonly serves as almost the sole illustration of the thermal quenching of luminescence from deep-level defects in numerous reviews, monographs, and textbooks, $3,20-26$

\section{B. Thermal quenching of PL in conductive semiconductors}

In $n$-type GaAs, several acceptors are responsible for the broad PL bands with maxima between 0.94 and $1.3 \mathrm{eV}$. Four of these bands were identified as different complexes involving vacancies and impurities. ${ }^{27}$ The quenching of these PL bands is attributed to the thermal emission of holes from the acceptors to the valence band. This mechanism is supported by a coherent rise of the near-band-edge PL intensity, in agreement with the rate-equation model discussed in Sec. II A and analyzed in detail in Refs. 1, 10, and 12.

The temperature dependencies of PL intensity for several defect-related PL bands in $n$-type and $p$-type GaP were studied by Gershenzon et al. ${ }^{28}$ The PL bands have been attributed to the DAP transitions involving different donors and acceptors such as $\mathrm{Te}_{\mathrm{P}}$ and $\mathrm{Ge}_{\mathrm{P}}$ (responsible for the $1.82 \mathrm{eV}$ band in $n$-type $\mathrm{GaP}$ ) or $\mathrm{Ge}_{\mathrm{Ga}}$ and $\mathrm{Zn}_{\mathrm{Ga}}$ (responsible for the $1.94 \mathrm{eV}$ band in $p$-type $\mathrm{GaP}$ ). The quenching of PL with temperature was attributed to thermal emission of holes from the shallow acceptor $\left(\mathrm{Ge}_{\mathrm{P}}\right)$ to the valence band in $n$ type $\mathrm{GaP}$ and to the emission of electrons from the shallow donor $\left(\mathrm{Ge}_{\mathrm{Ga}}\right)$ to the conduction band in $p$-type GaP. From the activation energies of the thermal quenching, the ionization energies for the corresponding donors and acceptors have been determined. In GaP, both donors and acceptors can be very shallow (e.g., $\mathrm{Te}_{\mathrm{P}}$ and $\mathrm{Zn}_{\mathrm{Ga}}$ with ionization energies smaller than $0.1 \mathrm{eV}) .{ }^{29}$ Then, the transitions of electrons from a shallow donor or the conduction band to an acceptor level in $n$-type $\mathrm{GaP}$ and the transitions of holes from a shallow acceptor or the valence band to a donor level in $p$-type $\mathrm{GaP}$ are both likely, and the temperature dependence of the corresponding PL can be described by the rate equations model presented in Sec. II A. However, in wide-bandgap semiconductors, such as $\mathrm{GaN}$ and $\mathrm{ZnO}$, the asymmetry between the shallow donors and acceptors $\left(E_{D} \ll E_{A}\right)$ leads to a situation where the dominant transitions in $n$-type and $p$-type are the same (from a shallow donor or the conduction band to different acceptor levels). ${ }^{6}$

The thermal quenching of the $1.45 \mathrm{eV}$ band in CdTe was analyzed in Refs. 30 and 31. This band is attributed to the DAP transitions involving a shallow donor (such as $\mathrm{I}_{\mathrm{Te}}$ or $\mathrm{In}_{\mathrm{Cd}}$ ) and the cadmium vacancy-shallow donor complex which is an acceptor (the so-called A center). In both works, the thermal quenching of the A center was explained with the Schön-Klasens mechanism. Interestingly, two activation energies were observed in the Arrhenius plot: $E_{1}=5-15 \mathrm{meV}$ and $E_{2}=95-125 \mathrm{meV}$. The smaller activation energy was attributed to thermal emission of electrons from a shallow donor to the conduction band, whereas the larger one was attributed to thermal emission of holes from the A center to the valence 
band. ${ }^{30,31}$ However, according to our model, ${ }^{1}$ the PL intensity via an acceptor in conductive $n$-type semiconductors depends only on the capture rates of holes by defects, and the thermal emission of electrons from the shallow donor should not affect the PL intensity if the PL band consists of unresolved $e A$ and DAP transitions. Since the concentration of free electrons was high in the studied samples $\left(1.3 \times 10^{18} \mathrm{~cm}^{-3}\right.$ in Ref. 30 and in the range of $10^{17}-10^{20} \mathrm{~cm}^{-3}$ in Ref. 31), we suggest that the decrease of PL intensity with the activation energy $E_{1}$ is associated with a weak temperature dependence of the capture coefficients (ignored in our model), rather than with emission of electrons to the conduction band.

In $\mathrm{ZnO}$, the most studied PL bands are the green luminescence (GL) band at $2.5 \mathrm{eV}$ attributed to an internal transition in $\mathrm{Cu}_{\mathrm{Zn}}{ }^{32}$ and the orange luminescence $(\mathrm{OL})$ band at $2.0 \mathrm{eV}$ attributed to the $\mathrm{Li}_{\mathrm{Zn}}$ acceptor. ${ }^{33}$ The ground state of the $\mathrm{Cu}_{\mathrm{Zn}}$ acceptor is close to the conduction band, whereas the PL arises due to the transition of a bound hole from an excited state (located at $\sim 0.4 \mathrm{eV}$ above the valence band maximum) to the ground state. ${ }^{32}$ The GL band in $n$-type $\mathrm{ZnO}$ is quenched with the activation energy $E_{A}=0.38 \mathrm{meV}$ at temperatures above $250 \mathrm{~K}^{34}$ The quenching is attributed to the thermal emission of holes from the excited state to the valence band. The quenching of the OL band in $n$-type $\mathrm{ZnO}$ at temperatures above $250 \mathrm{~K}$ reveals the activation energy of $E_{A}=0.5 \mathrm{eV} .{ }^{12,35}$ In agreement with our model, the thermal quenching of the OL band in samples with a very high IQE causes a stepwise rise of the near-bandedge emission intensity. ${ }^{12}$ Such behavior confirms that the quenching occurs via the Schön-Klasens mechanism.

In $n$-type GaN, thermal quenching of PL for many defects was studied and explained. ${ }^{6}$ One of the classical examples is the blue luminescence (BL) band related to the $\mathrm{Zn}_{\mathrm{Ga}}$ acceptor. In conductive $n$-type $\mathrm{GaN}$, the $\mathrm{BL}$ band is quenched with an activation energy of $0.35 \mathrm{eV}$, in agreement with the ionization energy of this acceptor. ${ }^{10}$ Figure 12 shows the temperature dependence of PL intensity for three $\mathrm{PL}$ bands in one of degenerate $\mathrm{GaN}: \mathrm{Si}, \mathrm{Zn}$ samples (sample 1142) studied in detail in Ref. 10. The BL band is quenched at $T>T_{0} \approx 300 \mathrm{~K}$. The temperature dependence of the $\mathrm{BL}$ band efficiency is fit with Eq. (8) where most of parameters are known from independent experiments. The parameter $E_{A}$ $(310 \mathrm{meV})$ is smaller than the ionization energy of the $\mathrm{Zn}_{\mathrm{Ga}}$ acceptor in undoped GaN (about $350 \mathrm{meV}$ ). ${ }^{6}$ The discrepancy can be explained by the bandgap renormalization in GaN heavily doped with $\left.\mathrm{Si}\left(n=10^{19} \mathrm{~cm}^{-3}\right)\right)^{36,37}$ The IQE of the BL band is very high in this sample $(\sim 90 \%)$. As a result, the quenching of the BL band causes significant rises for the other PL bands (Fig. 12). This fact proves that the quenching occurs via the Schön-Klasens (multi-center) mechanism.

The rise of PL intensity due to the quenching of some other recombination channels is a very typical phenomenon for PL in GaN and $\mathrm{ZnO}{ }^{6,38}$ In the model analyzed in Sec. II, we assumed that the dominant nonradiative defect has a very deep level, so that the thermal emission of electrons or holes from this level is negligible for the entire region of temperatures studied. Moreover, we assumed that the capture coefficients of the nonradiative defect (as well as for other defects) are temperature-independent. When nonradiative

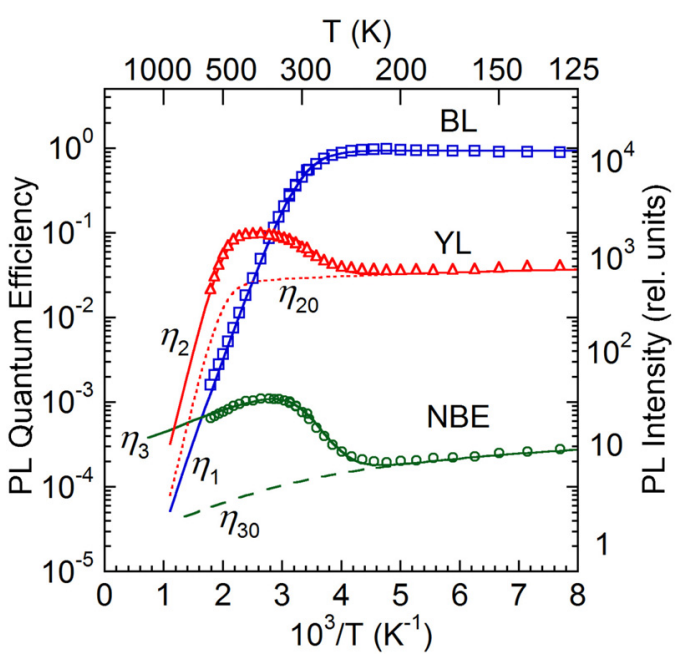

FIG. 12. Temperature dependence of the absolute IQE of PL in GaN:Si,Zn (sample 1142) at $P_{\text {exc }}=0.0015 \mathrm{~W} / \mathrm{cm}^{2}$. Points are experimental data. Solid blue curve shows $\eta_{1}$ calculated by using Eq. (8) with the following parameters: $\eta_{10}=0.96, g=2, C_{p A}=7 \times 10^{-7} \mathrm{~cm}^{3} / \mathrm{s}, \tau_{1}=0.55 \mu \mathrm{s}, E_{A 1}=310$ $\mathrm{meV}$. Dotted red and dashed green lines show $\eta_{20}(T)$ and $\eta_{30}(T)$ for the YL band and the NBE band, respectively, expected without the quenching of the BL band. Solid red and green lines show $\eta_{2}(T)$ and $\eta_{3}(T)$ calculated by using Eq. (11) with $\eta_{10}=0.92$ and $\eta_{10}=0.75$, respectively.

recombination is the dominant recombination mechanism, it is easy to verify the latter assumption. Indeed, the temperature dependence of $C_{p S}$ would cause the temperature dependence of $\eta_{0}$ [Eq. (9)], and then we would see the corresponding temperature dependence of PL intensity for all PL bands at $T<T_{0}$, because at these temperatures $\eta=\eta_{0}$. If recombination via the dominant nonradiative defect is "quenched" due to the thermal emission of charge carriers to the nearest band, we should see a simultaneous rise of PL intensity for all PL bands. We observed a rise of intensities for two of the PL bands with increasing temperature in Si-doped GaN and attributed it to the thermal quenching of the dominant nonradiative center with an activation energy of $155 \mathrm{meV} .^{12}$ The rise of PL intensity with increasing temperature has been observed for different semiconductors. ${ }^{15,39-44}$ This phenomenon is sometimes called the negative thermal quenching and is explained with a formalism similar to our model. ${ }^{44}$ Note that the assumptions made in Ref. 44 (such as that $N_{A}^{0}$ is independent of temperature, the rise of PL with temperature is caused by the thermal emission of electrons from the acceptor level to the conduction band, and the PL quenching is caused by the exponential increase of the nonradiative recombination rate with temperature) are not justified. However, if one assumes that the thermal emission of electrons or holes takes place from the dominant nonradiative defect to the nearest band, by using the rate equation model analyzed in Sec. II, most of the observed cases of the negative PL quenching can be naturally explained.

\section{Abrupt and tunable quenching of PL in high-resistivity $p$-type and insulating semiconductors}

\section{Thermal quenching of PL in Mg-and Zn-doped GaN}

The abrupt and tunable thermal quenching of defectrelated PL was observed in $\mathrm{Zn}$-doped and $\mathrm{Mg}$-doped 


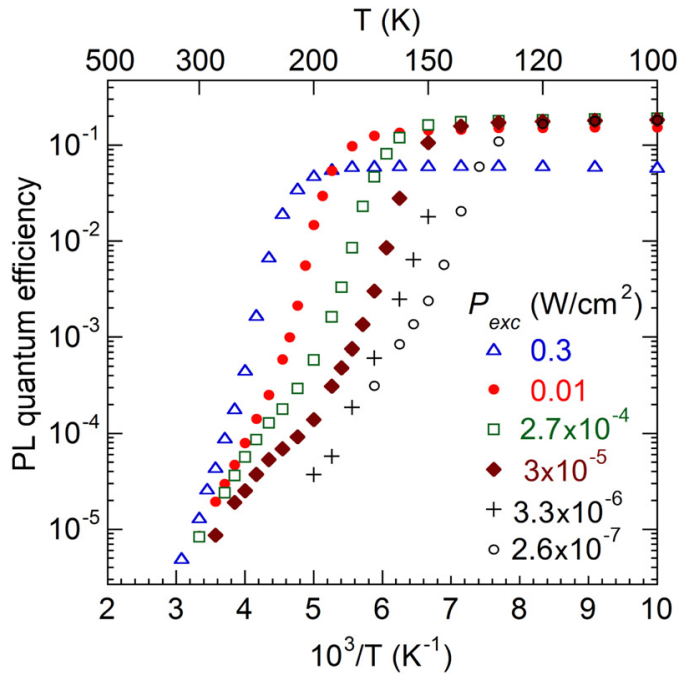

FIG. 13. Temperature dependence of quantum efficiency of the BL band in Zn-doped GaN (sample ap274) for excitation power densities between $2.6 \times$ $10^{-7}$ and $0.3 \mathrm{~W} / \mathrm{cm}^{2}$.

GaN. ${ }^{13,45,46}$ Temperature dependencies of the BL band intensity at several excitation intensities are shown in Fig. 13 for one of the high-resistivity Zn-doped samples. The $4.8 \mu \mathrm{m}$ thick GaN layer (sample ap274) has a concentration of $\mathrm{Zn}$ atoms of about $2 \times 10^{17} \mathrm{~cm}^{-3}$, as determined from secondary-ion mass-spectrometry. ${ }^{13}$ The intensity of the BL band drops at $T \approx T_{0}$, and the characteristic temperature of the abrupt quenching $T_{0}$ increases from 130 to $216 \mathrm{~K}$ as the excitation power density increases from $3 \times 10^{-7}$ to $0.3 \mathrm{~W} / \mathrm{cm}^{2}$. Similar temperature dependencies were observed for several other samples with a concentration of $\mathrm{Zn}$ between $\sim 10^{18}$ and $6 \times 10^{19} \mathrm{~cm}^{-3}$ and explained quantitatively with the phenomenological model discussed in Sec. II B. ${ }^{13}$ It appears that the dominant nonradiative center in the high-resistivity GaN:Zn layers is a deep donor with high capture cross-sections for both holes and electrons. The capture coefficients $C_{p S}$ and $C_{n S}$ for this defect were estimated to be on the order of $10^{-6}$ and $10^{-8}-10^{-7} \mathrm{~cm}^{3} / \mathrm{s}$, respectively. ${ }^{13,45}$

For one of the GaN:Zn samples ( $p$-type layer s560), a very unusual temperature dependence of the BL band intensity was observed (Fig. 14). The BL band is quenched in two steps. The characteristic temperatures of the first and second steps, $T_{1}$ and $T_{2}$, increase with increasing excitation intensity. The dependencies are explained within the same model, with the difference that a shallow acceptor with an ionization energy of about $0.2 \mathrm{eV}$ is included in the rate equations. ${ }^{45}$ This addition is justified by the fact that the intensity of the ultraviolet luminescence (UVL) band (attributed to the shallow acceptor) was the strongest in this sample among eight high-resistivity GaN:Zn samples. The first step of the PL quenching is caused by the thermal emission of holes from the shallow acceptor to the valence band and their re-capture at a nonradiative center and the deeper $\mathrm{Zn}_{\mathrm{Ga}}$ acceptor. The second drop in PL intensity is caused by the thermal emission of holes from the $\mathrm{Zn}_{\mathrm{Ga}}$ acceptor and their re-capture by the dominant nonradiative defect.

The tunable quenching of the UVL band in Mg-doped p-type $\mathrm{GaN}$ was reported in Ref. 46. The characteristic temperature $T_{0}$ increased from 80 to $130 \mathrm{~K}$ as the excitation

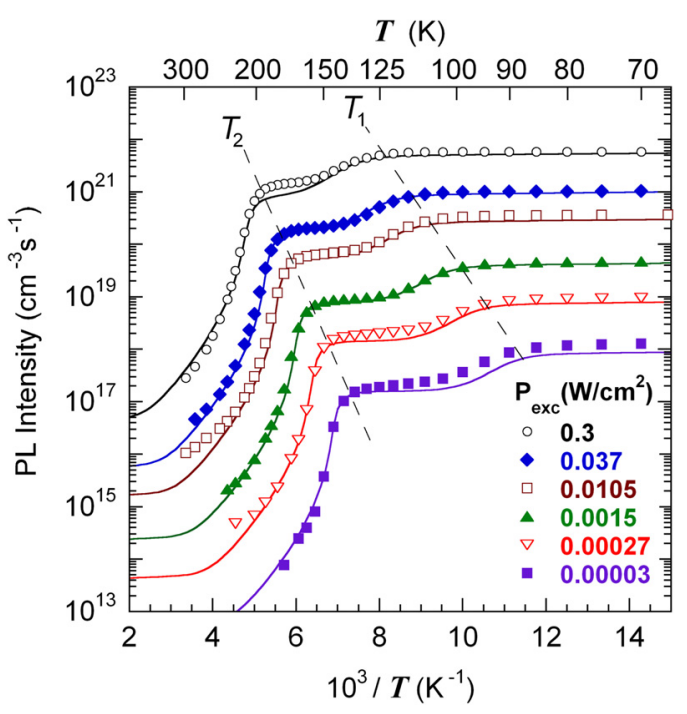

FIG. 14. Temperature dependence of PL intensity for the BL band in Zndoped GaN (sample s560) at selected excitation intensities. Solid curves are calculated using a model with four types of defects. Dashed lines show schematically the shift of characteristic temperatures $T_{1}$ and $T_{2}$ for different excitation intensities. (M. A. Reshchikov, Phys. Rev. B 85, 245203 (2012). Copyright 2012 American Physical Society.)

power density increased from $4 \times 10^{-5}$ to $24 \mathrm{~W} / \mathrm{cm}^{2}$. The drop of the PL intensity at $T_{0}$ is explained by the thermal emission of holes for the shallow $\mathrm{Mg}_{\mathrm{Ga}}$ acceptor $\left(E_{A} \approx 0.2\right.$ $\mathrm{eV})$ to the valence band and their subsequent capture by nonradiative centers. As a result, the nonradiative centers become mostly emptied of electrons at $T>T_{0}$, while at $T<T_{0}$ they were saturated with electrons. The drop in PL intensity is not as large and abrupt as for the BL band in Zn-doped GaN samples. However, this agrees with predictions of our theory for the shallower acceptor [Fig. 9(b)]. Interestingly, the tunable quenching was observed only for some $\mathrm{Mg}$-doped GaN samples and not observed for others. ${ }^{46}$ In the latter, the UVL band is quenched with very low activation energy in a wide temperature range. The difference in PL behaviors between the two groups of samples is attributed preliminarily to the existence of two types of nonradiative centers with different carrier-capture characteristics.

The dependence of the characteristic temperature $T_{0}$ on the electron-hole generation rate for selected $\mathrm{GaN}$ samples demonstrating the abrupt and tunable thermal quenching of PL is shown in Fig. 15. In addition to the data for the Zndoped and Mg-doped GaN samples, we included preliminary results for a high-resistivity undoped GaN sample. ${ }^{47}$ The dependencies of $T_{0}{ }^{-1}$ on $G$ represent straight lines, in agreement with Eq. (16). The slopes of the lines give the ionization energy of the acceptor which emits holes to the valence band and causes the sudden transition in the system from the population inversion at $T<T_{0}$ to the quasi-equilibrium at $T>T_{0}$. It appears that different shallow acceptors are responsible for the UVL band in undoped and Mg-doped GaN. This preliminary conclusion agrees with the findings of Monemar et al. ${ }^{48}$

\section{Tunable quenching of PL in other semiconductors}

In early works on phosphors, tunable quenching of PL has been reported. In particular, Klasens, ${ }^{5}$ Garlick and 


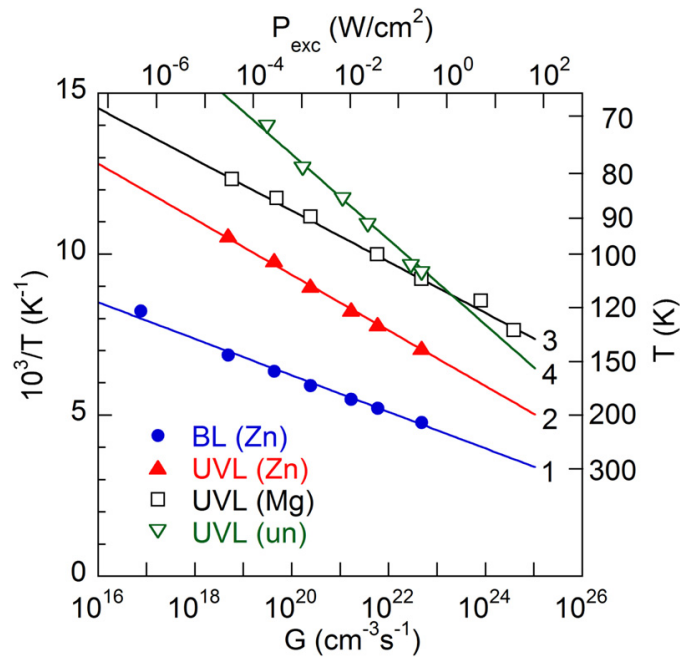

FIG. 15. Dependence of the characteristic temperature of abrupt quenching of PL on excitation intensity for insulating and $p$-type GaN samples. BL( $\mathrm{Zn})$ and UVL $(\mathrm{Zn})$ are the data for the BL band and UVL band, respectively, in p-type GaN doped with $\mathrm{Zn}$ [Ref. 45]; UVL(Mg) is the data for the UVL band in $p$-type GaN doped with Mg [Ref. 46]; UVL(un) is the data for the UVL band in insulating undoped GaN [Ref. 47]. Lines are calculated by using Eq. (16) with the following parameters: $B\left(\mathrm{~cm}^{-3} \mathrm{~s}^{-1}\right)$ and $E_{A}$ are $1 \times 10^{31}$ and $0.35 \mathrm{eV}$ (BL in GaN: $\mathrm{Zn}$ ), $7 \times 10^{30}$ and $0.23 \mathrm{eV}$ (UVL in GaN:Zn), $2 \times 10^{34}$ and $0.25 \mathrm{eV}$ (UVL in $\mathrm{GaN}: \mathrm{Mg}$ ), and $8 \times 10^{29}$ and $0.15 \mathrm{eV}$ (UVL in undoped GaN).

Gibson, ${ }^{49}$ and Vergunas and Gavrilov, ${ }^{50}$ reported on PL quenching from defects in $\mathrm{ZnS}$, which started at a characteristic temperature $T_{0}$. The value of $T_{0}$ increased with increasing excitation intensity or with decreasing concentration of killer centers (nonradiative defects) in $\mathrm{ZnS} .^{5,49,50}$ Both effects can be explained with our model [see Eqs. (16) and (17)]. This model also predicts a nonlinear dependence of PL intensity on excitation intensity in temperature range corresponding to PL quenching. ${ }^{51}$ Remarkably, already early studies of PL from phosphors and photoconductors ( $\mathrm{ZnS}$ and $\mathrm{ZnCdS}$ ) in $1930 \mathrm{~s}-1950 \mathrm{~s}$ reported on a superlinear increase of PL intensity with $G$ that was commonly described with a power law $I^{P L} \propto G^{m}$ with various powers $m$, exceeding 1 and sometimes $2 .^{52-58}$ An important feature of this unusual PL behavior is tunability of the effect by temperature. Specifically, the region of the nonlinear increase of PL efficiency shifts to higher $G$ with increasing temperature or with increasing concentration of PL "killer centers" (centers of nonradiative recombination). ${ }^{54,55,57}$

However, in early works, the researchers tried to explain the data by using Eq. (1) with a formal assumption that parameter $C$ depends on $G .^{49,59,60}$ Note that in all these works, $E_{A}$ in Eq. (1) was attributed to the ionization energy of a defect, while in our model for $p$-type semiconductors, an abrupt drop of PL intensity is predicted with the slope having no relation to the ionization energy of any defect. ${ }^{13}$

Maeda ${ }^{61,62}$ reported on tunable quenching of PL caused by DAP transitions in GaP and CdS. Similar to our results for high-resistivity GaN:Zn (Fig. 13), Maeda observed thermal quenching of PL above a characteristic temperature $T_{0}$, which increased with excitation intensity. Interestingly, for $\mathrm{CdS}$, the photoconductivity also decreased above the same $T_{0}$, and the tunable quenching was observed only in insulating samples.

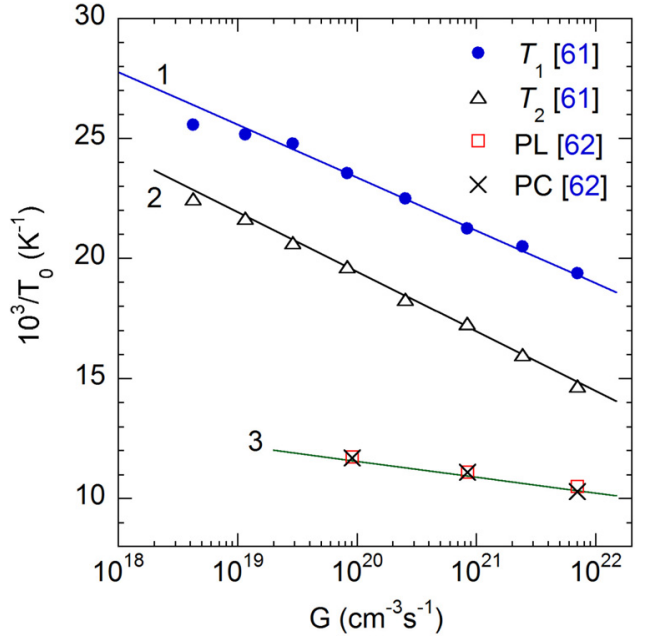

FIG. 16. Dependence of the characteristic temperature of abrupt quenching of PL on excitation intensity for insulating GaP and CdS samples studied in Refs. 61 and 62 . " $T_{1}$ " and " $T_{2}$ " are the data from Ref. 61 for two "break points" in the temperature dependence of PL intensity from insulating GaP. "PL" and "PC" are the data from Ref. 62 for the "break point" in the temperature dependence of PL intensity and photoconductivity, respectively, from insulating CdS. Solid lines are calculated by using Eq. (16) with the following parameters: $B\left(\mathrm{~cm}^{-3} \mathrm{~s}^{-1}\right)$ and $E_{A}$ are $4 \times 10^{30}$ and $0.09 \mathrm{eV}$ (line 1 for $T_{1}$ in PL from GaP [Ref. 61]), $7 \times 10^{27}$ and $0.08 \mathrm{eV}$ (line 2 for $T_{2}$ in PL from $\mathrm{GaP}$ [Ref. 61]), $3 \times 10^{37}$ and $0.3 \mathrm{eV}$ (line 1 for PL and photoconductivity in CdS [Ref. 62]).

Figure 16 shows the temperature dependencies of $T_{0}$ extracted from the experimental data of Maeda. ${ }^{61,62}$ For PL in $\mathrm{GaP}$, we included two sets of characteristic temperatures ( $T_{1}$ and $T_{2}$ ) because the quenching in Ref. 61 was not smooth but consisted of three regions with different activation energies, and the break points between these regions are denoted as $T_{1}$ and $T_{2}$ in Fig. 16. For PL in GaP, Maeda calculated the largest activation energy as $0.118 \mathrm{eV}$ from the slope in the Arrhenius plot. ${ }^{61}$ However, the slope in the dependence shown in Fig. 16 and calculated using Eq. (16) corresponds to the ionization energy of $0.08-0.09 \mathrm{eV}$ for an acceptor that causes the PL quenching. As for the data for $\mathrm{CdS}$, we calculated $E_{A}=0.3 \mathrm{eV}$ by fitting the dependence of $T_{0}$ on $G$ with Eq. (16). This is larger than the energy calculated by Maeda for the slope of thermal quenching $(0.165 \mathrm{eV})$. However, as we discussed in Ref. 13, the slope of the quenching can be affected by several reasons, among which potential fluctuations in insulating semiconductors appear to be the most important. $^{63}$

\section{About the Seitz-Mott mechanism of PL quenching}

The one-center or Seitz-Mott mechanism of PL quenching involves only one type of defect, which converts from radiative to nonradiative with increasing temperature due to certain properties of its adiabatic potentials. In general, it is difficult to choose between the Seitz-Mott and SchönKlasens mechanisms. Sometimes, it is possible to find reliably the position of the zero-phonon line (ZPL), either by direct observation of a sharp line in the high-energy part of a PL spectrum or from comparison of the PL and PL excitation (PLE) spectra. In this case, the ionization energy is expected to be close to the difference between the bandgap and the 
ZPL. If the activation energy in the thermal quenching process is close to this value, it is reasonable to attribute the quenching to the Schön-Klasens mechanism (the emission of holes to the valence band in $n$-type or electrons to the conduction band in $p$-type). Examples of such identification of the quenching mechanism include a number of defects in CdTe, ${ }^{30,31} \mathrm{ZnSe}^{64} \mathrm{GaAs}^{27}{ }^{27} \mathrm{GaP},{ }^{28}$ and $\mathrm{GaN} .{ }^{6}$ In the latter case, the activation energy of the quenching nearly coincides with the ionization energies of acceptors responsible for the YL (peak at $2.2 \mathrm{eV}), \mathrm{BL}(2.9 \mathrm{eV})$, and BL2 $(3.0 \mathrm{eV})$ bands. The attribution becomes unambiguous if simultaneously with the quenching of one PL band the intensities of all other PL bands rise. ${ }^{10}$ However, the quantum efficiency of the PL is often very low due to presence of nonradiative centers, and the rise may not be noticed.

In early works on PL from defects, attribution of the thermal quenching to the Seitz-Mott mechanism was very common and not always justified. For example, in a classic work by Hwang, ${ }^{18}$ the quenching of the $1.37 \mathrm{eV}$ band in $\mathrm{Zn}$ or Cd-doped $p$-type GaAs, which occurred at $T>70 \mathrm{~K}$ with an activation energy of $0.087 \mathrm{eV}$, was explained in terms of the Seitz-Mott mechanism. However, from comparison of the PL and PLE spectra obtained in that work, the ZPL line is expected to be located at $1.44 \mathrm{eV}$, about $0.08 \mathrm{eV}$ below the bandgap of GaAs at $20 \mathrm{~K}$. The main argument why Hwang chose the Seitz-Mott mechanism was the absence of the increase in the bandedge emission intensity simultaneously with the quenching. The possibility that nonradiative centers were the dominant recombination channel in these samples was not considered in this work. A similar analysis of the PL quenching behavior was carried out by Pal and Bose, ${ }^{65}$ who explained the quenching of the $\mathrm{Cu}$-related $1.21 \mathrm{eV}$ band in InP with the Seitz-Mott mechanism. Again, from the shape of the PL spectrum, we can estimate that the ZPL for this PL band is about $0.1 \mathrm{eV}$ below the bandgap edge. On the other hand, the accuracy of the activation energy in the PL quenching region $(77.4 \mathrm{meV})$ appears to be overestimated because the slope with such energy stretched for only one order of magnitude. Besides, the activation energy of the thermal quenching at about $100 \mathrm{~K}$ may be smaller than the ionization energy at $10 \mathrm{~K}$ because the acceptor level may move closer to the valence band with increasing temperature. Thus, the Schön-Klasens mechanism may be more appropriate for this case as well.

An instructive example of confusing the two mechanisms of the PL quenching is the self-activated PL in GaAs with the peak at $1.2 \mathrm{eV}$ that is attributed to the gallium vacancy-shallow donor $\left(\mathrm{V}_{\mathrm{Ga}} \mathrm{D}\right)$ complexes. ${ }^{20}$ Williams ${ }^{66}$ explained the temperature dependence of the $1.2 \mathrm{eV}$ PL intensity by the Seitz-Mott mechanism and attributed the activation energy of $0.18 \mathrm{eV}$ to a barrier between the excited and ground state of the luminescence center. Later, however, Glinchuk and Prokhorovich ${ }^{9,27}$ observed the characteristic competition between the recombination channels and unambiguously proved that the PL quenching occurs via the Schön-Klasens mechanism for the $\mathrm{V}_{\mathrm{Ga}} \mathrm{D}$ acceptors.

Among many defects in GaN, we found only two PL bands as candidates for the quenching with the Seitz-Mott mechanism. These are the RL2 band at $1.85 \mathrm{eV}$ and the GL2 band at $2.35 \mathrm{eV}$ in high-resistivity, very Ga-rich GaN. ${ }^{6}$ From comparison of the PL and PLE spectra for these bands, the ZPL is expected between 2.1 and $2.7 \mathrm{eV}$ for the RL2 band and between 2.7 and $3.4 \mathrm{eV}$ for the GL2 band, which would give the corresponding defect energy levels as counted from the valence band maximum. Thus, at least the RL2 band belongs to a deep defect. However, thermal quenching of PL for the RL2 and GL2 bands occurred with the activation energy of about $0.10-0.14 \mathrm{eV}$. Moreover, for both the RL2 and GL2 bands, PL after a pulsed excitation decayed exponentially, contrary to the nonexponential PL decay for majority of defects in GaN, which are attributed to DAP-type recombination at low temperature. ${ }^{6} \mathrm{We}$ speculated that some internal transitions in defects are responsible for such unusual behavior of PL. ${ }^{6}$ It is possible that the PL quenching for these defects occurs via the Seitz-Mott mechanism.

\section{CONCLUSION}

We analyzed the temperature dependencies of defectrelated PL intensity in GaN and few other semiconductors. A phenomenological model with three to four types of major point defects in a semiconductor is able to explain general trends in temperature behavior of PL in many cases, including conductive and insulating materials. For high-resistivity $p$-type and insulating semiconductors, nonlinear behavior of temperature dependencies is observed in experiments and explained using the above model. Two mechanisms of thermal quenching of PL, namely the Seitz-Mott mechanism and the Schön-Klasens mechanism, are analyzed in detail. We conclude that for majority of defects in III-V and II-VI semiconductors the thermal quenching of defect-related PL occurs via the Schön-Klasens mechanism, which involves thermal emission of charge carriers to the nearest band and redistribution of these carriers between all other recombination channels. Role of nonradiative defects in PL is analyzed. For high-resistivity semiconductors, we suggest a third mechanism of PL quenching, namely the abrupt and tunable thermal quenching. This kind of PL quenching can be observed when the dominant nonradiative defect has high capture cross-sections for both electrons and holes.

\section{ACKNOWLEDGMENTS}

The author wishes to thank Joy McNamara for technical assistance.

\footnotetext{
${ }^{1}$ M. A. Reshchikov and R. Y. Korotkov, Phys. Rev. B 64, 115205 (2001).

${ }^{2}$ A. M. Stoneham, Theory of Defects in Solids (Clarendon Press, Oxford, 2001). ISBN 0198507801.

${ }^{3}$ C. C. Klick and J. H. Schulman [in Solid State Physics, edited by F. Seitz and D. Turnbull (Academic Press Inc., New York, 1957), Vol. 5, p. 97].

${ }^{4}$ M. Schön, Z. Phys. 119, 463 (1942).

${ }^{5}$ H. A. Klasens, Nature 158, 306 (1946).

${ }^{6}$ M. A. Reshchikov and H. Morkoç, J. Appl. Phys. 97, 061301 (2005).

${ }^{7}$ F. Seitz, Trans. Faraday Soc. 35, 74 (1939); F. Seitz, Modern Theory of Solids (McGraw-Hill, New York, 1940).

${ }^{8}$ R. W. Gurney and N. F. Mott, Trans. Faraday Soc. 35, 69 (1939); N. F. Mott and R. W. Gurney, Electronic Processes in Ionic Crystals (Oxford University Press, London, 1948).

${ }^{9}$ V. I. Vovnenko, K. D. Glinchuk, and A. V. Prokhorovich, Sov. Phys. Semicond. 10, 320 (1976).
} 
${ }^{10}$ M. A. Reshchikov, M. A. Foussekis, J. D. McNamara, A. Behrends, A. Bakin, and A. Waag, J. Appl. Phys. 111, 073106 (2012).

${ }^{11}$ M. A. Reshchikov, G.-C. Yi, and B. W. Wessels, Phys. Rev. B 59, 13176 (1999).

${ }^{12}$ M. A. Reshchikov, "Internal Quantum Efficiency of Photoluminescence in Wide-Bandgap Semiconductors," in Photoluminescence: Applications, types and efficacy, edited by M. A. Case and B. C. Stout (Nova Science Publishers, Inc., New York), pp. 53-120, 2012, ISBN: 978-1-61942-426-5.

${ }^{13}$ M. A. Reshchikov, A. Kvasov, T. McMullen, M. F. Bishop, A. Usikov, V. Soukhoveev, and V. A. Dmitriev, Phys. Rev. B 84, 075212 (2011).

${ }^{14}$ C. G. Van de Walle and J. Neugebauer, J. Appl. Phys. 95, 3851 (2004).

${ }^{15}$ F. E. Williams and H. Eyring, J. Chem Phys. 15, 289 (1947); P. D. Johnson and F. E. Williams, J. Chem. Phys. 20, 124 (1952).

${ }^{16}$ C. C. Klick, Phys. Rev. 85, 154 (1952); D. L. Dexter, C. C. Klick, and G. A. Russel, Phys. Rev. 100, 603 (1955).

${ }^{17}$ H. P. de la Garanderie and D. Curie, "On the Theory of Thermal Quenching of Luminescence," in Luminescence of organic and inorganic materials, edited by H. P. Kallmann and G. M. Spruch (John Wiley and Sons Inc., New York, 1962), pp. 334-343.

${ }^{18}$ C. J. Hwang, Phys. Rev. 180, 827 (1969).

${ }^{19}$ D. Curie, Luminescence in Crystals (Methuen, London, 1963).

${ }^{20} \mathrm{E}$. W. Williams and H. B. Bebb, "Photoluminescence II: Gallium Arsenide," in Semiconductors and semimetals, edited by R. K. Willardson and A. C. Beer (Academic Press, New York, 1972), Vol. 8, pp. 321-392.

${ }^{21}$ S. Shionoya, "Photoluminescence," in Luminescence of solids, edited by D. R. Vij (Plenum Press, New York, 1998), pp. 95-133.

${ }^{22}$ J. I. Pankove, Optical Properties in Semiconductors (Dover Publ. Inc., New York, 1971), pp. 165-167.

${ }^{23}$ B. K. Ridley, Quantum Processes in Semiconductors, 4th ed. (Clarendon Press, Oxford 1999), pp. 235-281.

${ }^{24}$ M. Jaros, Deep Levels in Semiconductors (Adam Hilger Ltd., Bristol, 1982), pp. 182-199.

${ }^{25}$ D. R. Vij and N. Singh, "Basic Principles of Luminescence and Related Properties," in Luminescence and related properties of II-VI semiconductors, edited by D. R. Vij and N. Singh (Nova Science Publ. Inc., Commack, NY, 1998), pp. 1-35.

${ }^{26}$ C. R. Ronda, "Emission and Excitation Mechanisms of Phosphors," in Luminescence: From theory to applications, edited by C. Ronda (WilleyVCH Verlag, 2008), pp. 1-34. (ISBN: 978-3-527-31402-7).

${ }^{27}$ K. D. Glinchuk and A. V. Prokhorovich, Phys. Status Solidi A 44, 777 (1977).

${ }^{28}$ M. Gershenzon, F. A. Trumbore, R. M. Mikulyak, and M. Kowalchik, J. Appl. Phys. 37, 486 (1966).

${ }^{29}$ A. A. Kopylov and A. N. Pikhtin, Solid State Commun. 26, 735 (1978).

${ }^{30}$ J. Lee, N. C. Giles, D. Rajavel, and C. J. Summers, J. Appl. Phys. 78, 5669 (1995).

${ }^{31}$ W. Stadler, D. M. Hofmann, H. C. Alt, T. Muschik, B. K. Meyer, E. Weigel, G. Müller-Vogt, M. Salk, E. Rupp, and K. W. Benz, Phys. Rev. B 51, 10619 (1995).

${ }^{32}$ R. Dingle, Phys. Rev. Lett. 23, 579 (1969).

${ }^{33}$ D. Zwingel, J. Lumin. 5, 385 (1972).
${ }^{34}$ M. A. Reshchikov, V. Avrutin, N. Izyumskaya, R. Shimada, H. Morkoç, and S. W. Novak, J. Vac. Sci. Technol. B 27, 1749 (2009).

${ }^{35}$ M. A. Reshchikov, J. Garbus, G. Lopez, M. Ruchala, B. Nemeth, and J. Nause, Mater. Res. Soc. Symp. Proc. 957, K07-19 (2007).

${ }^{36}$ M. Yoshikawa, M. Kunzer, J. Wagner, H. Obloh, P. Schlotter, R. Schmidt, N. Herres, and U. Kaufmann, J. Appl. Phys. 86, 4400 (1999).

${ }^{37}$ A. Walsh, J. L. F. Da Silva, and S.-H. Wei, Phys. Rev. B 78, 075211 (2008).

${ }^{38}$ M. A. Reshchikov, X. Gu, J. Nause, and H. Morkoç, Mater. Res. Soc. Symp. Proc. 892, FF23.11 (2006).

${ }^{39}$ M. Z. Cieplak and M. Godlewski, J. Appl. Phys. 60, 4259 (1986).

${ }^{40}$ S. S. Kurbanov, G. N. Panin, T. W. Kim, and T. W. Kang, J. Lumin. 129, 1099 (2009).

${ }^{41}$ D. M. Hofmann, B. K. Meyer, H. Alves, F. Leiter, W. Burkhard, N. Romanov, Y. Kim, J. Krüger, and E. R. Weber, Phys. Status Solidi A 180, 261 (2000).

${ }^{42}$ M. Koós, I. Kósa Somogyi, and V. A. Vassilyev, J. Lumin. 26, 449 (1982).

${ }^{43}$ H. He, Y. Wang, J. Wang, and Z. Ye, Phys. Chem. Chem. Phys. 13, 14902 (2011).

${ }^{44}$ H. Shibata, Jpn. J. Appl. Phys., Part 1 37, 550 (1998).

${ }^{45}$ M. A. Reshchikov, Phys. Rev. B 85, 245203 (2012).

${ }^{46}$ M. A. Reshchikov, J. McNamara, S. Fernandez, and R. Calarco, Phys. Rev. B 87, 115205 (2013).

${ }^{47}$ M. A. Reshchikov, J. D. McNamara, and F. Shahedipour-Sandvik (unpublished).

${ }^{48}$ B. Monemar, P. P. Paskov, G. Pozina, C. Hemmingsson, J. P. Bergman, T. Kawashima, H. Amano, I. Akasaki, T. Paskova, S. Figge, D. Hommel, and A. Usui, Phys. Rev. Lett. 102, 235501 (2009).

${ }^{49}$ G. F. J. Garlick and A. F. Gibson, J. Opt. Soc. Am. 39, 935 (1949).

${ }^{50}$ F. I. Vergunas and F. F. Gavrilov, Z. Exp. Theor. Fiz. 20, 224 (1950) [in Russian].

${ }^{51}$ M. A. Reshchikov, A. J. Olsen, M. F. Bishop, and T. McMullen, Phys. Rev. B 88, 075204 (2013).

${ }^{52}$ N. Riehl, Z. Tech. Phys. 20, 152 (1939).

${ }^{53}$ J. H. Gisolf and F. A. Kröger, Physica 6, 1101 (1939).

${ }^{54}$ F. Urbach, A. Urbach, and M. Schwartz, J. Opt. Soc. Am. 37, 122 (1947).

${ }^{55}$ N. R. Nail, F. Urbach, and D. Pearlman, J. Opt. Soc. Am. 39, 690 (1949).

${ }^{56}$ F. I. Vergunas and F. F. Gavrilov, Z. Exp. Theor. Fiz. 18, 873 (1948) [in Russian].

${ }^{57}$ M. N. Alentsev, V. V. Antonov-Romanovsky, and L. A. Vinokurov, Dokl. Akad. Nauk SSSR 96, 1133 (1954) [in Russian].

${ }^{58}$ P. S. Litvinova, Z. Exp. Theor. Fiz. 19, 688 (1949) [in Russian].

${ }^{59}$ M. E. Wise and H. A. Klasens, J. Opt. Soc. Am. 38, 226 (1948).

${ }^{60}$ R. H. Bube, Phys. Rev. 90, 70 (1953).

${ }^{61}$ K. Maeda, J. Phys. Chem. Solids 26, 595 (1965).

${ }^{62}$ K. Maeda, J. Phys. Chem. Solids 26, 1419 (1965).

${ }^{63}$ B. I. Shklovskii and A. L. Efros, Electronic Properties of Doped Semiconductors (Springer, Berlin, 1984).

${ }^{64}$ P. J. Dean, B. J. Fitzpatrick, and R. N. Bhargava, Phys. Rev. B 26, 2016 (1982).

${ }^{65}$ D. Pal and D. N. Bose, J. Appl. Phys. 78, 5206 (1995).

${ }^{66}$ E. W. Williams, Phys. Rev. 168, 922 (1968). 